\title{
Brain-based ranking of cognitive domains to predict schizophrenia
}

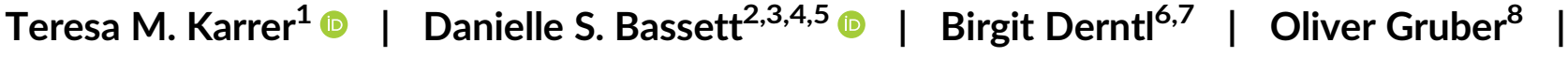 \\ André Aleman $^{9}$ | Renaud Jardri ${ }^{10}$ | Angela R. Laird ${ }^{11}$ | Peter T. Fox ${ }^{12,13,14}$ | \\ Simon B. Eickhoff ${ }^{15,16}$ | Olivier Grisel ${ }^{17}$ | Gaël Varoquaux ${ }^{17}$ | Bertrand Thirion ${ }^{17}$ \\ Danilo Bzdok ${ }^{1,6,17}$
}

\footnotetext{
${ }^{1}$ Department of Psychiatry, Psychotherapy and Psychosomatics, Faculty of Medicine, RWTH Aachen, Aachen, Germany

${ }^{2}$ Department of Bioengineering, University of Pennsylvania, Philadelphia, Pennsylvania

${ }^{3}$ Department of Electrical and Systems Engineering, University of Pennsylvania, Philadelphia, Pennsylvania

${ }^{4}$ Department of Neurology, Hospital of the University of Pennsylvania, Philadelphia, Pennsylvania

${ }^{5}$ Department of Physics and Astronomy, University of Pennsylvania, Philadelphia, Pennsylvania

${ }^{6}$ Translational Brain Medicine, Jülich Aachen Research Alliance (JARA), Aachen, Germany

${ }^{7}$ Department of Psychiatry and Psychotherapy, University of Tübingen, Tübingen, Germany

${ }^{8}$ Department of Psychiatry, University of Heidelberg, Heidelberg, Germany

${ }^{9} \mathrm{BCN}$ Neuroimaging Center, University Medical Center Groningen, University of Groningen, Groningen, The Netherlands

${ }^{10}$ Division of Psychiatry, University of Lille, CNRS UMR 9193, SCALab and CHU Lille, Fontan Hospital, Lille, France

${ }^{11}$ Department of Physics, Florida International University, Miami, Florida

${ }^{12}$ Research Imaging Institute, University of Texas Health Science Center, San Antonio, Texas

${ }^{13}$ South Texas Veterans Health Care System, San Antonio, Texas

${ }^{14}$ State Key Laboratory for Brain and Cognitive Sciences, University of Hong Kong, Hong Kong, China

${ }^{15}$ Institute of Systems Neuroscience, Heinrich-Heine University, Düsseldorf, Germany

${ }^{16}$ Institute of Neuroscience and Medicine (INM-7), Research Centre Jülich, Jülich, Germany

${ }^{17}$ Parietal Team, INRIA Saclay/NeuroSpin, Palaiseau, France
}

Correspondence

Danilo Bzdok, Department of Psychiatry, Psychotherapy and Psychosomatics, Faculty of Medicine, Universitätsklinikum Aachen, Pauwelsstr. 30, 52074 Aachen, Germany. Email: danilo.bzdok@rwth-aachen.de

Funding information

Deutsche Forschungsgemeinschaft, Grant/ Award Numbers: BZ2/2-1, BZ2/3-1, BZ2/4-1; Paul Allen Foundation; John D. and Catherine T. MacArthur Foundation; Alfred P. Sloan Foundation; ISI Foundation; Exploratory Research Space, Grant/Award Number: OPSF449; START-Program of the Faculty of Medicine, Grant/Award Number: 126/16; Amazon AWS Research Grant; International Research Training Group, Grant/Award Number: IRTG2150

\begin{abstract}
Schizophrenia is a devastating brain disorder that disturbs sensory perception, motor action, and abstract thought. Its clinical phenotype implies dysfunction of various mental domains, which has motivated a series of theories regarding the underlying pathophysiology. Aiming at a predictive benchmark of a catalog of cognitive functions, we developed a data-driven machine-learning strategy and provide a proof of principle in a multisite clinical dataset $(n=324)$. Existing neuroscientific knowledge on diverse cognitive domains was first condensed into neurotopographical maps. We then examined how the ensuing meta-analytic cognitive priors can distinguish patients and controls using brain morphology and intrinsic functional connectivity. Some affected cognitive domains supported well-studied directions of research on auditory evaluation and social cognition. However, rarely suspected cognitive domains also emerged as disease relevant, including self-oriented processing of bodily
\end{abstract}


sensations in gustation and pain. Such algorithmic charting of the cognitive landscape can be used to make targeted recommendations for future mental health research.

\section{KEYWORDS}

BrainMap database, coordinate-based meta-analysis, ontology of the mind, pattern recognition, predictive analytics, statistical learning

\section{1 | INTRODUCTION}

Schizophrenia is among the most severe mental disorders but has so far evaded mechanistic understanding. This major psychiatric disorder affects $\sim 1 \%$ of the world population (McGrath, Saha, Chant, \& Welham, 2008) and presents a long-enduring clinical course in many patients (Hegarty, Baldessarini, Tohen, Waternaux, \& Oepen, 1994), including social and occupational dysfunctions (Tandon et al., 2013). The associated economic costs per year range between $\$ 94$ million and $\$ 102$ billion per country (Chong et al., 2016). Schizophrenia thus imposes a huge burden on the affected individuals, their families, and society at large (Charlson et al., 2018; Wittchen et al., 2011). To eventually improve clinical care and intervention, it will be instructive to systematically explore the nature of the disease.

The clinical presentations of schizophrenia strongly suggest various cognitive impairments ranging from basic sensory perception, motor action, affective response to higher order cognition, and social interaction (Javitt \& Freedman, 2015; Taylor et al., 2012; Tost \& MeyerLindenberg, 2012). The advent of in vivo neuroimaging has enabled the investigation of the neural basis of these cognitive functions and their aberrations in disease. For more than 20 years now, functional neuroimaging experiments have accumulated hints about the candidate disease processes in schizophrenia, including, for instance, impaired auditory change detection (Erickson, Ruffle, \& Gold, 2016; Umbricht \& Krljes, 2005), emotional face recognition (Kohler, Walker, Martin, Healey, \& Moberg, 2010; Li, Chan, McAlonan, \& Gong, 2010), and working memory (Forbes, Carrick, McIntosh, \& Lawrie, 2009; Schneider et al., 2007). Yet, today, it is still incompletely understood "where schizophrenia is located in the brain" (Dhindsa \& Goldstein, 2016; Elert, 2014; Sullivan, 2012; Weinberger \& Radulescu, 2016).

Carefully designed experimental studies require that the participants attend to and execute the presented tasks for extended periods of time. The maintenance of controlled cognitive sets has sometimes been challenging to ascertain in psychiatric patients (Eickhoff \& Etkin, 2016; Weinberger \& Radulescu, 2016). Fortunately, mounting evidence suggests that many of the characteristic neural activity patterns described during defined experimental tasks have some correspondence in neural activity observed during task-free resting-state scanning (Bzdok et al., 2016; Cole, Bassett, Power, Braver, \& Petersen, 2014; Smith et al., 2009; Tavor et al., 2016). Therefore, responseindependent brain scans in clinical populations might provide unprecedented insights into brain systems dedicated to different mental operations. Additionally, despite many successes, experiments in patients with schizophrenia that test hypotheses regarding cognitive processes can carefully probe only a limited number of brain systems at a time. Such circumscribed research efforts could be complemented by computational modeling approaches that simultaneously inspect a diverse collection of cognitive functions.

The heterogeneous clinical picture of schizophrenia patients lends itself particularly well to take a step back and impartially test diverse cognitive functions for their relevance in schizophrenia. To derive a brain-informed ranking of cognitive processes implicated in schizophrenia, we integrated existing neuroscientific knowledge on cognitive processes into a new machine learning pipeline. For this purpose, we capitalized on an established description system of cognitive processes and a multisite dataset of structural and functional brain scans. The cognitive taxonomy has previously been used to systematically annotate roughly a quarter of the published neuroimaging experiments (Derrfuss \& Mar, 2009). We quantitatively summarized this large body of evidence on the functional basis of diverse cognitive processes using coordinate-based meta-analyses. For each particular cognitive process, we computed the typically activated functional network, henceforth "cognitive meta-prior." To evaluate the cognitive meta-priors for their usefulness in predicting which brain scan belongs to a schizophrenia patient, we developed a novel machine-learning approach.

In a first step, we built cognitive domain-specific base models to distinguish between schizophrenia patients and healthy controls. The cognitive meta-priors guided the extraction of information from structural and functional brain data of the schizophrenia dataset. That is, each base model offers interpretability by extracting structural and functional brain information according to how a particular cognitive process maps to the brain. In a second step, we combined the cognitive domain-specific models into a higher level model that puts all cognitive meta-priors of varying cortical and subcortical spread on a comparable scale. The integration into a summary model (using "stacking", cf. below) enabled us to benchmark diverse cognitive processes for their importance in schizophrenia. The ranking of cognitive meta-priors was based on combined neurobiological information from brain structure and function to increase the generality of our results. In the entire process, we relied on minimal pathophysiological, neurobiological, and statistical assumptions. In sum, we automatically computed, validated, and ranked a catalog of cognitive processes for their relative impairment in schizophrenia. 


\section{2 | METHODS}

\section{1 | Cognitive description system: BrainMap taxonomy}

The cognitive science community has not yet agreed on a consensus definition for mental operations (cf. Fox et al., 2005; Poldrack \& Yarkoni, 2016). Among other possibilities, the BrainMap initiative provides an established means to describe the repertoire of mental operations (Fox \& Lancaster, 1994). Experts have steadily refined the description system over two decades. Today, it is one of the most frequently applied taxonomies in research practice (Fox \& Lancaster, 2002; Laird, Eickhoff, Kurth, et al., 2009). In particular, BrainMap offers two distinct taxonomies to categorize mental operations: (a) mental domains that span sensory, motor, affective, and higher level cognitive processes that are recruited during psychological paradigms (i.e., "behavioral domains") and (b) the types of experimental tasks used to evoke cognitive processes of interest in a controlled fashion (i.e., "paradigm classes") (Laird, Eickhoff, Li, et al., 2009). Both taxonomies have been used to systematically annotate $>16,000$ archived neuroimaging experiments from peer-reviewed publications (Fox \& Lancaster, 2002; Laird, Eickhoff, et al., 2011). The completeness and correctness of the labeling of the neuroimaging experiments has been verified by several members of the BrainMap team. Taken together, BrainMap offers the unique combination of a systematic cognitive taxonomy, designed and refined by authorities in their fields, and its consistent application to a large repository of existing neuroimaging studies.

The present study capitalized on both description systems to increase the chances of identifying the most pertinent brain-behavior mappings in schizophrenia. To avoid conceptual overlap between the psychological categories considered within each taxonomy, we removed the hierarchical dependence between mental domains by excluding any top-level classes. For example, we excluded "emotion" as an overarching category, and instead considered the subordinates "disgust," "fear," "happiness," and "sadness." We also disregarded rarely used cognitive concepts, defined as those with less than 50 functional neuroimaging experiments in the BrainMap database. By considering only cognitive domains that can be based on a sufficient number of neuroimaging experiments (Bossier et al., 2018; Eickhoff et al., 2016), we could construct robust and meaningful brain-behavior maps, as we will describe in detail in the next section. A final set of 34 mental domains (Figure 1) and 50 experimental tasks (Figures 1 and S1) from BrainMap was submitted to a computational approach to test for their utility in schizophrenia prediction.

\section{2 | Constructing cognitive meta-priors in healthy participants: Activation likelihood estimation meta-analysis of BrainMap taxonomy}

We carried out quantitative meta-analyses to synthesize existing neurobiological knowledge across tens of thousands of neuroimaging experiments from healthy individuals. For each particular cognitive domain, we derived one whole-brain signature of neural activity changes by using coordinate-based meta-analysis. The widely used activation likelihood estimation (ALE) approach summarized the peak activations reported by functional imaging experiments (Eickhoff et al., 2009; Eickhoff, Bzdok, Laird, Kurth, \& Fox, 2012; Turkeltaub et al., 2012). ALE meta-analysis treated the reported coordinates of significant experimental neural response as centers of threedimensional probability distributions that capture the spatial uncertainty of neuroimaging results (Eickhoff et al., 2009; Eickhoff et al., 2012; Turkeltaub et al., 2012). The spatial extent of the Gaussian probability distribution incorporated empirical estimates of betweentemplate and between-participant variance of neuroimaging peaks (Eickhoff et al., 2009). For each BrainMap experiment, the probability distributions of the reported peak coordinates were merged into a modeled activation map. The use of a nonadditive approach prevented local summation effects (Turkeltaub et al., 2012). Finally, all activation maps associated with a particular cognitive process were united to a probability map. The resulting ALE scores yielded the probability of increased neural activity measured during a particular experimental study for each gray-matter voxel. Since ALE scores are influenced by the number of experiments that they are based on, the meta-analytic networks were $z$-scored by mean centering to zero and unit-variance scaling to one. This normalization step of each meta-analytic network aimed at improving the comparability between different cognitive meta-priors. Thus, we quantitatively summarized the consistent topography of neural activity engagements pertaining to each cognitive category of a taxonomy.

\section{3 | Clinical brain-imaging resources: Multisite schizophrenia cohort}

Given the well-documented diversity of schizophrenia symptoms, we evaluated the cognitive meta-priors in a high number of patients from several psychiatric hospitals. We capitalized on a five-site imaging dataset that provided brain scans from patients with schizophrenia and matched healthy controls $(n=428)$. Written informed consent for study participation was obtained from all participants. The data acquisition was approved by the ethics committees of the universities of Aachen, Albuquerque, Göttingen, Utrecht, and Lille. All patients were diagnosed by board-certified psychiatrists according to international statistical classification system of diseases and related health problems-10 or diagnostic and statistical manual of mental disorders-IV-TR criteria. In healthy controls, any history of neurological or psychiatric disorders was ruled out via structured clinical interview. The dataset included (a) demographic indicators including age and sex, (b) structural brain-imaging data (sMRI), and (c) resting-state functional brain-imaging data (fMRI). All behavioral and brain-imaging information was anonymized. The sMRI and fMRI data were acquired on common 3T scanners (see Table S1 for details). Preprocessing of the imaging data was performed in SPM8 (Statistical Parametric Mapping, Wellcome Department of Imaging Neuroscience, London, UK, http:// www.fil.ion.ucl.ac.uk/spm/) using MATLAB R2014a (MathWorks, Natick, MA). In our analyses, we included only those participants for 


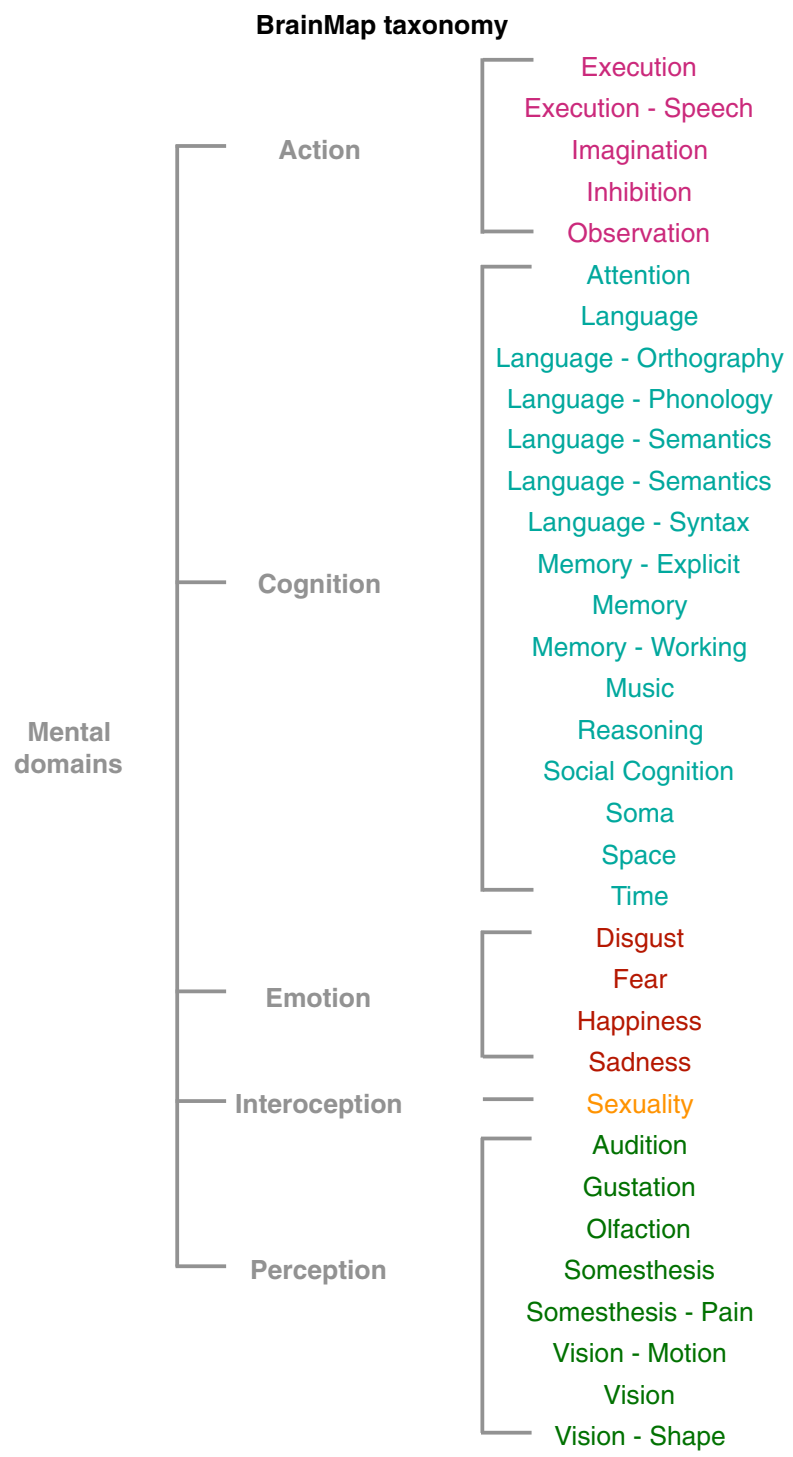

\section{Exemplary cognitive meta-priors}
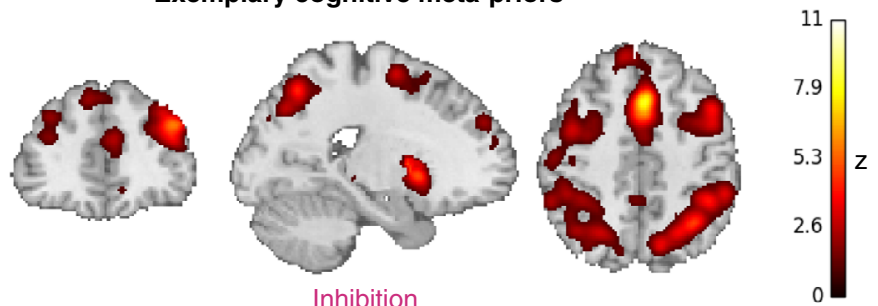

Inhibition
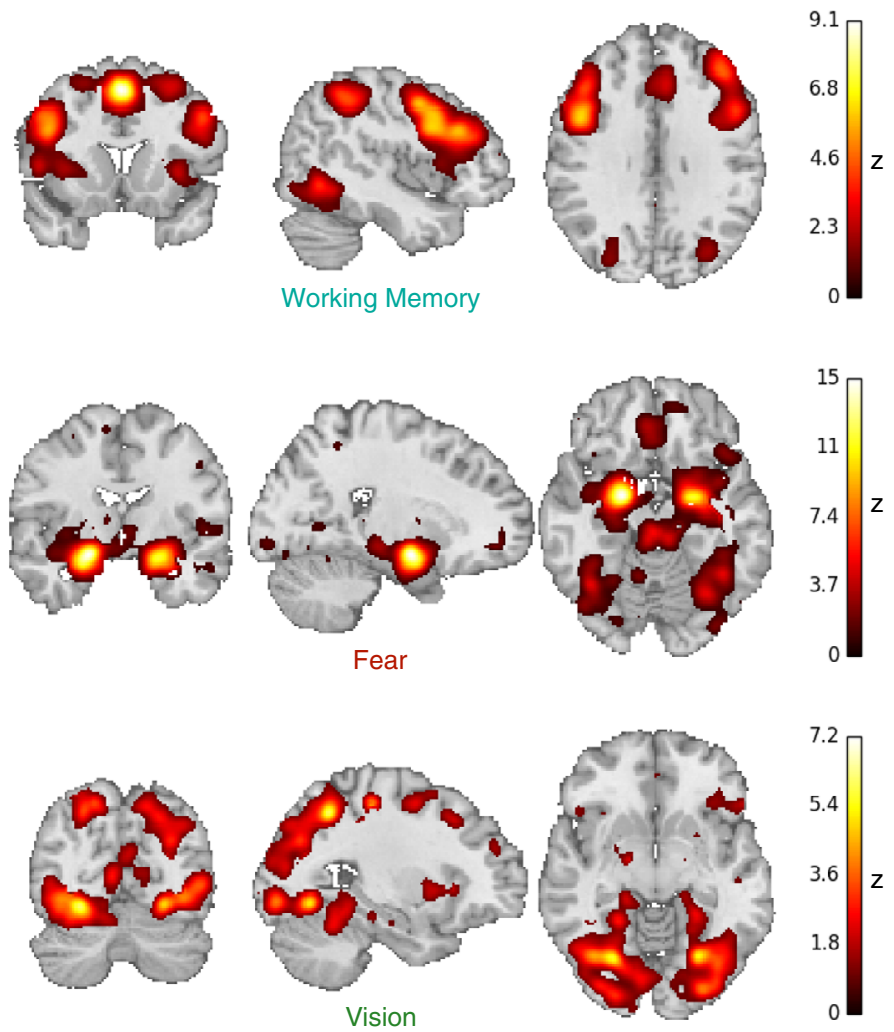

FIGURE 1 Overview of a taxonomy that compartmentalizes human cognition. (Left) Exhaustive set of mental operations used for braindriven ranking of altered cognitive concepts in schizophrenia. BrainMap defines two description systems: Mental domains (shown here) and experimental tasks (Figure S1). Note that the five top classes (action, etc.) were disregarded in the present study to avoid hierarchical dependence between the cognitive classes. This database offers results of almost a quarter of the published functional neuroimaging experiments carefully annotated with both taxonomies. (Right) A cognition-topography map for each cognitive category was generated from the neuroimaging database. Four examples of cognitive meta-priors are shown (z-scored for display, only voxels with positive z-scores shown) [Color figure can be viewed at wileyonlinelibrary.com]

whom both sMRI and $\mathrm{fMRI}$ data were available. This step enabled us to jointly examine neurobiological impairment in brain structure and brain function. The final sample of 161 patients and 163 healthy controls presented the basis for our machine-learning workflow (see Table 1 for sample characteristics). The total 324 participants were matched for age and sex, both within and across sites (Table 1).

\section{4 | Brain structure: Voxel-based morphometry}

To investigate how brain anatomy in healthy controls deviates from brain anatomy in patients with schizophrenia, we relied on volume information in T1-weighted brain scans (Table S1). The preprocessing of the whole-brain morphometric maps was performed using standard settings in the VBM8 toolbox (https://dbm.neuro.uni-jena.de/vbm). The anatomical maps were spatially normalized to MNI space (ICBM-152 template) using the DARTEL toolbox including both affine and nonlinear spatial transformation. We then quantified the probability of each voxel to belong to gray matter, white matter, and cerebrospinal fluid to segment the volumetric brain maps into the three tissue types. To remedy bias-field inhomogeneities, we applied a unified segmentation (Ashburner \& Friston, 2005). Partial volume correction was carried out to account for blurring into neighboring voxels. Furthermore, nonlinear modulation adjusted for interindividual volumetric differences during the warping process to $\mathrm{MNI}$ space. In this way, we obtained gray-matter volume measures for each participant that were corrected for individual brain size. Additionally, we accounted for potential confounding effects 
TABLE 1 Clinical sample from different sites

\begin{tabular}{|c|c|c|c|c|}
\hline Sample & $n$ (males) & $\begin{array}{l}\text { Sex } \\
\text { differences } \\
\left(^{(p \text { values })^{a}}\right.\end{array}$ & $\begin{array}{l}\text { Age } \\
\text { (years) }\end{array}$ & $\begin{array}{l}\text { Age } \\
\text { differences } \\
\text { ( } p \text { values) }^{b}\end{array}$ \\
\hline \multicolumn{5}{|c|}{ Groningen } \\
\hline $\mathrm{SCZ}$ & $32(19)$ & & $33.6 \pm 11.1$ & \\
\hline $\mathrm{HC}$ & $32(19)$ & 0.476 & $31.6 \pm 11.2$ & 1 \\
\hline \multicolumn{5}{|c|}{ Göttingen } \\
\hline SCZ & $32(26)$ & & $32.3 \pm 9.9$ & \\
\hline $\mathrm{HC}$ & $29(22)$ & 0.889 & $31.9 \pm 9.4$ & 0.841 \\
\hline \multicolumn{5}{|l|}{ Aachen } \\
\hline SCZ & $14(11)$ & & $35.1 \pm 11.1$ & \\
\hline $\mathrm{HC}$ & $13(10)$ & 0.682 & $33.2 \pm 12.0$ & 0.719 \\
\hline \multicolumn{5}{|l|}{ Lille } \\
\hline SCZ & $15(9)$ & & $33.3 \pm 5.0$ & \\
\hline $\mathrm{HC}$ & $16(11)$ & 0.048 & $29.0 \pm 6.3$ & 0.894 \\
\hline \multicolumn{5}{|l|}{ COBRE } \\
\hline SCZ & $68(55)$ & & $38.2 \pm 13.7$ & \\
\hline $\mathrm{HC}$ & $73(50)$ & 0.270 & $35.8 \pm 11.7$ & 0.136 \\
\hline \multicolumn{5}{|c|}{ Total analyzed sample } \\
\hline $\mathrm{SCZ}$ & $161(120)$ & & $35.4 \pm 11.9$ & \\
\hline $\mathrm{HC}$ & $163(112)$ & 0.125 & $33.4 \pm 11.0$ & 0.299 \\
\hline
\end{tabular}

Note. Patients with $\mathrm{SCZ}$ and matched $\mathrm{HC}$; age values in mean $\pm \mathrm{SD}$. Abbreviations: HC, healthy control; SCZ, schizophrenia.

${ }^{a}$ Statistical comparison of age differences between groups performed via $t$ test;

${ }^{\mathrm{b}}$ Statistical comparison of sex differences between groups performed via chi-squared test.

of age, sex, and site to discourage the predictive algorithms from picking up these influences of no interest.

\section{5 | Brain function: Intrinsic resting-state connectivity}

To examine group differences in intrinsic neural activity, we relied on fMRI maps acquired by resting-state echo-planar imaging (Table S1). Before recording of the task-independent blood oxigenation level dependent (BOLD) signal scans, the participants were asked to lie still in the scanner and let their minds wander without thinking of anything in particular. Adherence to the instructions was verified in postscan interviews. The first four MRI scans of each participant were discarded to account for magnetic field saturation. The resting-state time series were aligned by a two-pass procedure to account for participant movements during the scanning session. After coregistration, the functional resting-state maps were spatially registered to $\mathrm{MNI}$ standard space (ICBM-152 template), analogous to the sMRI scans. The resulting brain maps were smoothed by a 12-mm full width at half maximum Gaussian kernel (Glahn et al., 2008; Laird, Fox, et al., 2011; Smith et al., 2009). To further account for potential confounding effects through head motion, we corrected the time series of each voxel by a common set of 24 motion parameters: (a) the six motion parameters extracted from image realignment, (b) their first derivatives, and (c) the respective squared terms of original motion parameters and derivatives. This specific motion correction procedure was found to improve ensuing functional connectivity analyses by yielding more specific and sensitive brain signals (Chai, Castanon, Ongur, \& Whitfield-Gabrieli, 2012; Satterthwaite et al., 2013). We did not apply global signal regression (Murphy, Birn, Handwerker, Jones, \& Bandettini, 2009; Yeh, Tseng, Lin, Tsai, \& Huang, 2015). The BOLD time series were band-pass filtered for frequencies between 0.01 and $0.08 \mathrm{~Hz}$ using the frequency-domain filter in the CONN toolbox (https://www.nitrc.org/projects/conn). This frequency range is commonly assumed to represent neural activity and to be less prone to physiological artifacts such as respiration and heart rate (Fox \& Raichle, 2007; Lu et al., 2007). Additionally, the BOLD signal time series of each voxel were converted to z-scores in each participant to allow for group analyses. At the across-participant level, we finally helped remove potential confounding influences by accounting for age, sex, and site differences in the fMRI data.

\subsection{Sampling neurobiological characteristics of the brain: Complementary data-extraction pipelines}

The derived cognitive meta-priors guided information extraction from structural and functional brain data by focusing on different neurobiological characteristics. We wished to relax a priori assumptions on the most relevant principle of brain organization in schizophrenia (Weinberger \& Radulescu, 2016). We therefore applied different sampling procedures to accommodate common approaches to aggregate brain data. These preprocessing steps ensured that the various cognitive meta-priors yielded the same number of variables in each pipeline to allow for statistical comparability in schizophrenia classification (Hastie, Tibshirani, \& Friedman, 2001). Otherwise, different model complexities could have made it difficult to attribute lack of predictability to either the brain data themselves or possible discrepancies of the modeling procedure.

Three overarching strategies profited from distinct and complementary ways to aggregate neurobiological information:

1. Mining peak locations: Our "peak activation" approaches concentrated on the most important voxel groups of a given cognitive meta-prior. Target voxels were extracted by searching for locations with the highest probability of increased neural activity during the engagement of a particular cognitive process. Hence, this simple strategy selected a subset of the most important gray-matter voxels from the brain maps guided by the meta-priors. Because the procedure was based on inspection of single voxels in structural or functional brain data, the analyses were perhaps closest, in character, to mass-univariate analyses prevalent in neuroimaging:

a. The "highest absolute activation peaks" approach sampled the voxels with the highest probability of increased neural activity from each cognitive meta-prior without imposing additional assumptions. The original meta-analytic maps of 
the corresponding neurocognitive priors were used to extract the voxels of generally largest signal changes.

b. The "highest specific activation peaks" approach sampled the peak voxels after accounting for voxels with high acrossdomain baseline activity by subtracting the mean neural activity level for each voxel across meta-priors. For instance, large-scale analyses showed regions of the saliency network and of the frontoparietal network to have the highest taskresponse probabilities (Nelson et al., 2010; Yarkoni, Poldrack, Nichols, Van Essen, \& Wager, 2011). The preference of voxels that were specifically increased in neural activity by a particular cognitive process tended to enhance the relative differences between meta-priors.

c. The "standardized activation peaks" approach accounted for both the mean and the variance of each voxel observed across meta-priors. Before identifying the target voxels, we subtracted the mean neural activity and scaled the voxels to unit variance across meta-priors.

2. Mining regional characteristics: An alternative procedure was deployed to acknowledge the perhaps still most dominant view on brain organization (Kanwisher, 2010; Passingham, Stephan, \& Kotter, 2002). Our "regional specialization" approaches followed the idea that the brain is partitioned into localized, nonoverlapping regions (cf. Finn et al., 2015; Glasser et al., 2015). The perspective emphasizes that cognitive processes may be realized by recruitment of neuronal populations that occur in disjoint brain compartments. Clustering methods naturally dovetail with grouping similar voxels into distinct brain regions (Eickhoff, Thirion, Varoquaux, \& Bzdok, 2015; Thirion, Varoquaux, Dohmatob, \& Poline, 2014) by assigning each voxel to exactly one brain region only. Three complementary clustering algorithms were used to merge voxels to homogeneous clusters such that the voxels within a region are more similar to each other than between regions:

a. K-means clustering iteratively readjusts the region centers and then reassigns the voxels to each nearest cluster center by minimizing the Euclidean distance of the voxels within each cluster (Lloyd, 1957; Nanetti, Cerliani, Gazzola, Renken, \& Keysers, 2009). The partitioning procedure relied on minimal assumptions and imposed, for instance, no spatial constraints so that the extracted regions were not necessarily spatially contiguous.

b. Ward clustering is a hierarchical clustering algorithm that successively combines the most similar voxels until a number of specified regions are reached. Ward clustering aims at minimizing the variance between voxels within each cluster (Johnson, 1967). In contrast to the more liberal constraints of k-means, only neighboring voxels were fused which resulted in spatially contiguous regions in the brain (Abraham et al., 2014).

c. Spectral clustering transforms the data in a nonlinear fashion, which complements the k-means and ward clustering approaches. The nonlinear transformation enabled the spectral clustering algorithms to discover nonconvex clusters that contrasted with those obtained with k-means and ward clustering.
First, a similarity graph was constructed that represented spatial proximity between the voxels (van Luxburg, 2007). Then, the graph was partitioned such that the weight of the edges cut was small compared to the weights of the edges inside each cluster (Donath \& Hofman, 1973; Thirion et al., 2014). Different from the k-means clustering approach and analogous to the ward clustering approach, only spatially contiguous voxels were merged into region clusters.

3. Mining network characteristics: Yet another complementary procedure accommodated the organizational perspective of brain function arising from an interplay of distributed, overlapping networks (cf. Smith et al., 2009). Our "distributed networks" approaches created network variables by focusing on the functional connections between distinct brain compartments that are cross-regionally integrated (Sporns, 2014; Van Essen, Anderson, \& Felleman, 1992). This conceptualization is naturally captured by matrix decomposition algorithms that broke down the brain into a number of hidden distributed network components (Smith et al., 2009). In contrast to the "regional specialization" approach, each voxel belonged to each of the components to varying degrees:

a. Principal component analysis (PCA) is a widespread procedure that searches for spatially uncorrelated network components that explain the observed variance distributed in the brain data (Shlens, 2014). The orthogonal components consisted of linear combinations of the voxels, while all gray-matter voxels were assigned continuously to each network and nonlinear relationships between the variables were ignored.

b. Sparse PCA is a recent variant of PCA that additionally exploits the fact that often only a subset of voxels is relevant for extracting coherent network components to explain most of the observed variance in the data (Zou, Hastie, \& Tibshirani, 2006). A sparse representation was accomplished by additionally imposing a parsimony constraint (L1 penalty) that also partly relaxed the orthogonality assumption of classical PCA (Chennubhotla \& Jepson, 2001).

c. Independent component analysis is able to discover the sources of variation that independently contributed to the observations in the brain, instead of imposing uncorrelatedness between networks such as in the PCA approaches (Calhoun, Adali, Pearlson, \& Pekar, 2001; Hvarinen, 1999). Complementing PCA, the neural signal was nonlinearly separated into network components where a particular network node could readily contribute to more than one network component (Hvarinen, 1999).

In sum, our data preparation pipelines sampled complementary aspects of brain biology by means of importantly different dimensionality-reduction techniques. This meta-prior-guided extraction of sMRI and fMRI data enabled direct comparison of our analytical approach in (a) brain structure, (b) brain function, and (c) their combination. For the joint analyses of both imaging modalities, we concatenated the extracted SMRI and fMRI data for each 
participant. In sum, nine complementary sampling pipelines extracted meaningful neurobiological information from patients and controls by guidance through the meta-priors and commonly used dimensionalityreduction techniques.

\section{7 | Confederating ensembles of cognitive meta-priors: Model stacking for integrated prediction}

For each cognitive meta-prior, we used the extracted brain information to train a predictive model (i.e., "base model") for disease status classification. The collection of base models was incorporated into one higher level predictive model (i.e., "composite model") to stratify the cognitive domains according to their prediction performance. This two-step stacking strategy (Breiman, 1996; Wolpert, 1992) put each meta-prior to a comparable scale and identified their relative relevance for schizophrenia classification, despite their naturally diverging neurobiological representations. By placing all meta-prior models on a common scale for each taxonomy, the summary model automatically ranked the whole set of cognitive processes according to their potential involvement in schizophrenia.

1. Base models: Separately for each cognitive category of a taxonomy, we fitted one simple linear classification model to disambiguate the groups based on the extracted and z-scored brain data. $z$-Scoring brings neurobiological variables with different distributions to a same comparable unit and thus, ensures their equal contribution in the subsequent modeling process (Gelman \& Hill, 2006). In analyses involving $\mathrm{fMRI}$, the 25th percentile of the highest scoring resting-state connectivity features was selected first (in the training data, cf. below) according to the strength of univariate relationships with the participant group. The adaption of this feature-selection step, similar to the sMRI analyses, was intended to improve comparability between both imaging modalities. For each cognitive meta-prior, we thus fitted a logisticregression algorithm to the extracted SMRI and fMRI data of a larger part of the participants (i.e., training sample). Then, we used the built logistic regression model to predict disease status (schizophrenia vs. health) in the previously left-out participants (i.e., test sample). The evaluation of disease status in new participants yielded practically relevant predictions because the algorithm did not visit the participants during model estimation (Bzdok, \& Ioannidis, 2019, Gabrieli, Ghosh, \& Whitfield-Gabrieli, 2015). Thus, the base models predicted the probability for a given participant to be affected by schizophrenia from the structural and functional brain data. The independent (probabilistic) disease status predictions of each cognitive meta-prior served as input for the integrated model.

2. Composite model: The meta-prior specific predictions of the base models were combined for training a more elaborate predictive model. The integrative model considered the separate relevances of all cognitive processes of a taxonomy at the same time for schizophrenia detection. For this purpose, we used a random forest algorithm because the classifier can capture complicated nonlinear relationships combined with the possibility of model interpretability. This pattern-learning algorithm involves fitting a collection of decorrelated decision trees and uses their majority vote for prediction (Breiman, 2001; Louppe, 2014). As a first advantage, the ensuing committee classifier was able to quantify the single meta-priors regarding their contribution for schizophrenia classification (Breiman, 2001; Louppe, Wehenkel, Sutera, \& Geurts, 2013). As an ensuing second advantage, random forests could uncover potential nonlinear interactions between the meta-priors, and thus their corresponding cognitive classes. Since we wished to reduce variability in the classification process, we set a common choice of trees in the random forest to 1,000 . The depth of the trees was set to 5 because higher order interactions between cognitive processes would have evaded ready visualization or interpretation. The maximum number of features considered at each split in a tree was set to 1 , which encouraged decorrelated trees and further improved the equal opportunity between the meta-priors. This analysis setting ensured that the composite model was only minimally affected by potential redundancy in the base models corresponding to specific meta-priors. Overall, the integration of these base models into a summary model (stacking) enabled us to rank each taxonomy of cognitive domains in their ability to distinguish between patients and controls.

\section{8 | Model evaluation: Nested 10 -fold cross- validation for single-participant prediction}

For the obtained cognitive domain-overarching predictive model, we estimate the capability to correctly distinguish brain data from participants that we would observe in the future, as an approximation of external validation. To examine the performance of the neurobiologically informed composite model in participants whom the algorithm has not seen before, we implemented a nested, stratified 10 -fold cross-validation. The participants were divided into 10 balanced data splits (folds), each preserving the percentage of participants of both classes. The predictive model was repeatedly fitted on $90 \%$ of the data and subsequently assessed in the brain-data of the left-out $10 \%$ of the participants (Hastie et al., 2001; Stone, 1974; Stone, 1978). After 10 iterations of model fitting and testing, the percentage of correctly classified test participants was averaged across folds. The nested variant of the cross-validation scheme ensured that only actual base model predictions were fed into the composite model as one important characteristic of stacking procedures (Hastie et al., 2001; Wolpert, 1992). Note that we aimed at validating the practical plausibility of our approach for interpretable single-patient prediction, instead of tuning our model toward highest-possible prediction accuracies. The obtained quantity yielded the cross-validated prediction accuracy of the integrative composite model to generalize to future participant samples from the population.

We additionally evaluated how much the obtained classification performances in other schizophrenic patients would be expected to vary. For this purpose, we computed their $95 \%$ population confidence intervals using bootstrapping (Bzdok, Nichols, \& Smith, 2019; Efron \& Tibshirani, 1994). The statistical procedure generates alternative datasets by repeatedly drawing random samples of the original data 
with replacement. In 1,000 bootstrap iterations, the identical nested crossvalidation scheme was carried out on the perturbed participant samples. This uncertainty interval estimation answered how the classification success was expected to vary in the broader schizophrenia population. The classification performance of the composite model enabled comparisons between (a) different imaging modalities (sMRI, fMRI, and combined sMRI and $\mathrm{fMRI}$ data), (b) the set of complementary procedures of neurobiological sampling, and (c) two BrainMap taxonomies (mental domains and experimental tasks). Furthermore, the model performance allowed us to validate the composite model against models not informed by cognitive meta-priors (cf. below). In sum, testing the generalizability of the composite model helped us gain confidence in the robustness and potential clinical usefulness of our data-analysis approach.

\section{9 | Model inspection: Variable importance, nonlinear effects, and predictive relevance maps}

We explored the predictive contribution of the individual meta-priors in a taxonomy in an identical process. Our stacking-model approach allowed reverse engineering which 34 mental domains or 50 experimental tasks might be most affected in schizophrenia. For the purpose of model interpretation, the composite model was initially refitted on the full participant sample (Hastie et al., 2001). Random forest algorithms naturally afford a quantitative measure of relative importance for each input variable (Breiman, 2001). Technically, the variable importance of a meta-prior provides a convenient summary of the mean decrease in the misclassification rate across all branch splits in which a specific variable was used in a grown decision tree to separate the healthy and schizophrenic group (Louppe et al., 2013). Since each input variable fed into the random forest corresponded to a single meta-prior, the variable importance of the composite model weighted the ensemble of cognitive domains in a same step. We capitalized on these relative importance weights to assign each meta-prior a ranking position according to its disease discriminability. The highest values of importance indicated the first rank. To quantitatively estimate the precision of the ranking positions of the meta-priors in the general population, we estimated the $95 \%$ confidence intervals in 1,000 bootstrap iterations by repeatedly fitting the final model to resampled alternative datasets. Since the model evaluation revealed that none of the nine pipelines were uniformly superior, we averaged the ranking positions across all of them to enhance impartiality of neurobiological assumptions. As an overall uncertainty estimate accounting for random sampling effects, the $95 \%$ confidence intervals were calculated from the bootstrapped distributions of the variable importances across pipelines. In addition to the relative contribution to schizophrenia, the random forest also allowed the investigation of potential nonlinear interactions between the cognitive meta-priors. As two-way interactions are easier to understand by humans than higher order interactions, we detailed the interaction surface for each pair of cognitive domains after accommodating the remaining cognitive metapriors contribution to the prediction of schizophrenia. To additionally capture discriminative characteristics of schizophrenia on the neurobiological level, we investigated which brain regions were most pertinent for disease classification. For this purpose, we multiplied each (z-scored) cognitive meta-prior with its respective importance weight of the composite model. Then, we averaged the ensuing maps corresponding to the cognitive domains to provide a global predictive relevance map for schizophrenia. Across neurobiological sampling pipelines, we thus inspected the predictive value of the cognitive domains, their interaction in disease classification, and their neurobiological basis.

\subsection{0 | Testing the cognitive specificity of schizophrenia predictability: Comparison to a null model}

A negative test ensured the fit for purpose of the final predictive model across imaging modalities (Kuhn \& Johnson, 2013). This sanity check answered the question "Did we successfully distinguish patients from controls because the summary model captured the individual configurations of cognitive facets rather than other characteristics of our participant sample?" To this end, we examined the null hypothesis that no coherent relation exists between the configuration of cognitive facets of healthy controls and schizophrenia patients. The placebo hypothesis was put to the test by a nonparametric permutation procedure (Efron, 2012; Winkler, Ridgway, Douaud, Nichols, \& Smith, 2016). We specifically corrupted cognition-related structure in the data, while leaving the other joint probabilities intact. That is, we only perturbed variance in the data related to the alternative hypothesis of individual expressions of cognitive meta-priors achieving disease classification. We randomly exchanged the importance of individual meta-priors between participants, separately in patients and controls, before they were fed into the composite model. This permutation scheme preserved the manner in which each cognitive meta-prior scored and the disease structure of our sample. Yet, the procedure was targeted at altering the participant-level pattern of meta-prior expressions. Put differently, the permutation changed how the meta-prior relevance co-occurred in combinations within patients and within controls. Based on these slightly permuted data, the same composite model was fit 1,000 times to compute a distribution of classification performances that occur under the null hypothesis. Subsequently, we compared the actually obtained prediction accuracy of our meta-model against the no-effect distribution. In each data analysis pipeline, the comparison of the composite model to the performance of a null model allowed us to ascertain that our disease classification was based on the combined cognitive facets in individual participants.

\subsection{1 | Scientific-computing implementation}

Our data-processing workflow was implemented in Python 2.7. We chose the open-source programming language to enable the reproducibility of our results and encourage reuse of our code in future projects. All computational analyses relied on unit-tested implementations of most recent machine-learning algorithms as provided by scikit-learn 18.1 (Pedregosa et al., 2011). The application of the predictive models to high-dimensional neuroimaging data was facilitated by nilearn 3.0 (Abraham et al., 2014). The full analysis workflow completed after $>7$ days on our computing 
cluster hosted at the Rechenzentrum of RWTH Aachen University with 52 cores and 512 GB working memory. All data analysis scripts are publicly available for transparency and reuse at https://github.com/ TMKarrer/domain_ranking_scz.

\section{3 | RESULTS}

\section{1 | Constructing cognitive meta-priors from a large-scale neuroimaging database}

We jointly screened a set of cognitive functions for their predictive value in schizophrenia. For this purpose, we capitalized on a large database of functional neuroimaging experiments that have been expert-labeled according to two complementary taxonomies (Figure 2a): mental domains (Figure 1) and experimental tasks (Figure S1). For each cognitive category of a taxonomy, we used quantitative meta-analyses to summarize the annotated functional neuroimaging findings observed in thousands of healthy individuals from the BrainMap database (Figure 2b). We generated whole-brain maps of robust neural activity changes for each of 34 mental domains and 50 experimental tasks. On average, the cognitive meta-priors underlying the mental domains quantitatively synthesized 265 (ranging from 50 to 1,123) database experiments, whereas the neurocognitive primitives associated with experimental tasks synthesized 167 (ranging

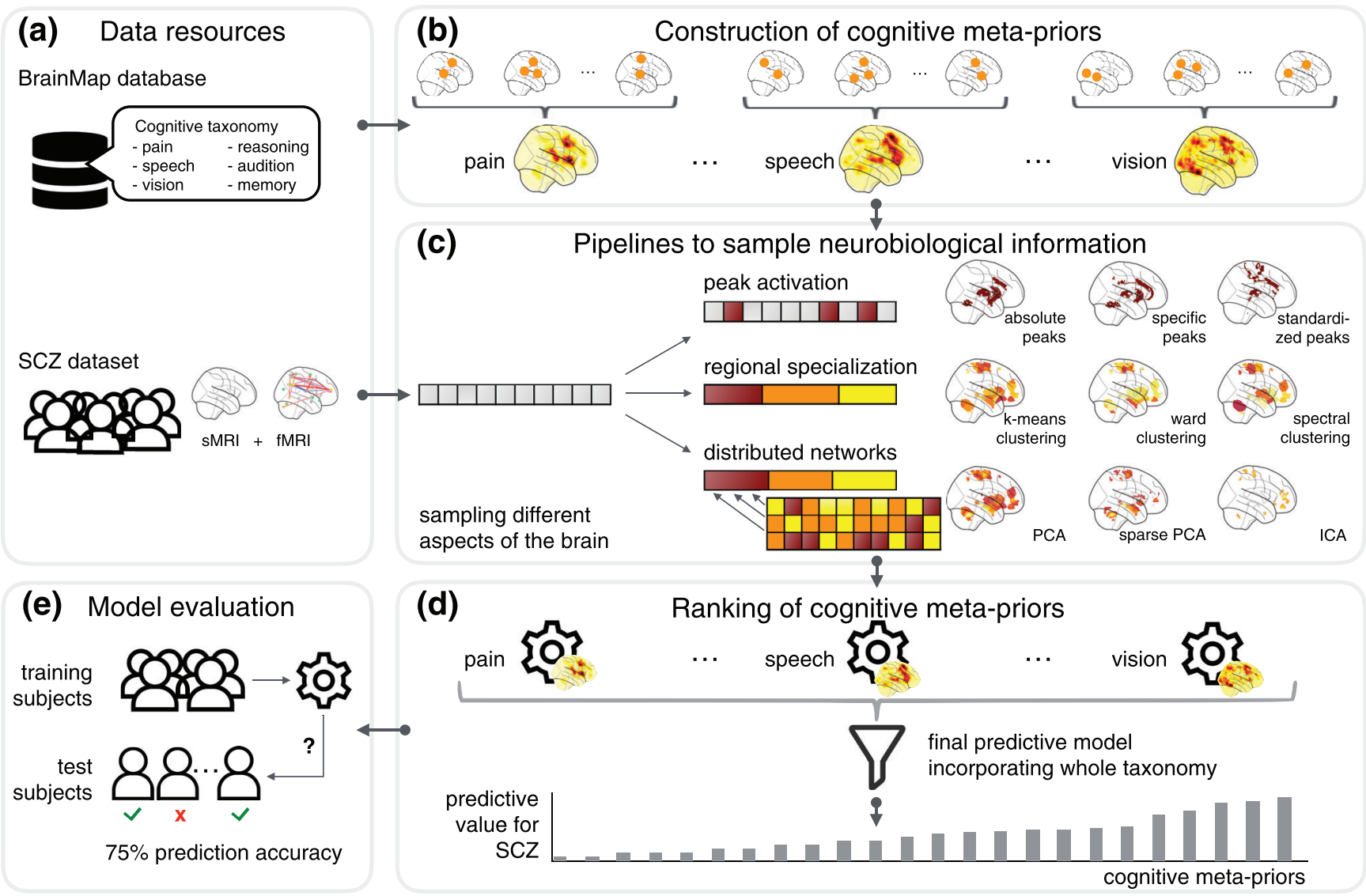

FIGURE 2 Overview of our analysis workflow. Illustrates our approach to automatically rank a set of commonly studied cognitive processes for their predictive relevance in schizophrenia (SCZ). (a) We capitalized on two types of data resources. The BrainMap database provided existing neuroscience knowledge in form of robust neural activity changes reported in published neuroimaging experiments. Each experiment was labeled with the examined cognitive processes by means of a comprehensive cognitive taxonomy. Additionally, we built on structural and functional magnetic resonance imaging data (sMRI and $\mathrm{FMRI}$ ) from a multisite dataset of patients with $\mathrm{SCZ}$ and healthy controls (HCs). (b) From the neuroimaging database, we quantitatively summarized the topography of consistently evoked neural activity changes associated with each cognitive domain (e.g., pain) into a "cognitive meta-prior." (c) The ensuing set of cognitive meta-priors served as masks to extract cognitive domain-specific information from structural and functional brain scans of SCZ patients and HCs. The data extraction followed nine complementary ways to aggregate neurobiological information, such as mining peak locations, local region, and integrative network characteristics. (d) To impartially rank the cognitive meta-priors for their predictive value in SCZ, we used a two-step approach. First, we built several base models to test each particular cognitive meta-prior separately for its capability of telling patients and controls apart. Second, we combined the collection of all base model predictions into a higher level summary model encapsulating the entire cognitive taxonomy. The summary model put all meta-priors on the same scale and could thus directly compare a variety of cognitive processes in their usefulness for detecting SCZ from brain scans. The resulting rankings of cognitive processes were averaged across the nine different neurobiological sampling strategies. (e) Finally, we validated the ability of the built predictive model to distinguish patients and controls in the future based on previously unseen, left-out participants (10-fold cross-validation scheme) [Color figure can be viewed at wileyonlinelibrary.com] 
from 50 to 701) neuroimaging studies in BrainMap (Tables S2 and S3). As expected, the cognitive concepts clearly differed in their spatially distributed set of responsive brain regions.

Regarding mental domains (Figure 1; see Figure S1 for experimental tasks), a database of studies linked to motor inhibition, for example, consistently engaged mainly the bilateral supplementary motor area and posterior insula, but also involved the putamen, frontal eye field, and intraparietal sulcus in most individuals. Working memory processes, instead, robustly recruited a distributed set of bilateral brain regions, including the dorsolateral frontal cortex and dorsal anterior cingulate cortex but also the inferior parietal lobe and precuneus. The neural responses coherently observed across experiences of fear were located in the bilateral amygdala, extending into the neighboring hippocampus as well as bilateral anterior cingulate cortex, and ventromedial frontal cortex and the right posterior insula. The neural activity pertaining to visual perception was prominent in the bilateral inferior and middle occipital gyri of the early visual cortex, extending into the fusiform gyri, and posterior parietal lobe, as well as middle and inferior temporal gyri (not shown). For both BrainMap taxonomies, the cognitive meta-priors encapsulated quantitatively dissociable patterns of whole-brain activity underlying a class of cognitive processes.

We made several general observations across the synthesis of neurocognitive priors underpinning mental domains and experimental tasks. Various cognitive processes related to basic perception corresponded to bilateral activity increases in primary sensory and association cortices and insula, but also mapped onto the putamen as well as superior and medial frontal gyri. Different cognitive processes associated with motor action primarily elicited bilateral activity increases in the precentral gyri and thalamus, and also recruited insula and medial frontal gyri. Emotion-related processes were mainly subserved by the bilateral amygdala and both anterior and posterior cingulate cortex but also frequently involved the medial prefrontal cortex. Many higher level cognitive processes tended to predominantly evoke increases in neural activity in bilateral prefrontal regions and posterior cingulate cortex, and further involved bilateral inferior and superior parietal lobe and middle temporal gyri. Across domains of both taxonomies, mental domains and experimental tasks, we observed that many cognitive functions were underpinned by a distributed constellation of higher and lower level brain systems.

\section{2 | Estimating model performance across imaging modalities and brain sampling approaches}

The cognitive meta-priors guided the extraction of structural (sMRI) and functional (fMRI) brain data in a five-site schizophrenia dataset ( $n=324$, mean age $=35.4 \pm 11.5$ years; Table 1$)$. We retrieved the neurobiological information using nine complementary data aggregation strategies that sampled neural activity changes (a) at peak locations, (b) in brain regions, and (c) distributed brain networks (Figure 2c). For each cognitive meta-prior, the thus aggregated brain information was used to build one dedicated predictive model. That is, each such base model was informed by one cognitive process when applied to the whole-brain sMRI and fMRI data to separate patients from controls. These domain-specific models exclusively learned from brain information that was linked to a particular cognitive process. The domain-specific models served as building blocks to form a domain-spanning predictive model (Figure $2 \mathrm{~d}$ ). This summary model put all cognitive meta-priors on a same scale, which is an important prerequisite to impartially rank them according to their relevance for schizophrenia. Initially, we wished to assess the ability of the summary model to predict disease status in new participants. For this purpose, we repeatedly tested the predictive model in previously left-out participants who were not seen by any predictive model before (10-fold cross-validation; Figure 2e). Across brain structure and function and complementary brain sampling tactics, the domain-integrating summary model performed consistently better than chance $(50 \%)$ in classifying new individuals (Figure S2).

We first compared the classification performance across imaging modalities (Figure S2). We observed that predictive models only informed by brain structure correctly predicted disease status in $73.2 \%$ ( $S D=2.1 \%$, across brain sampling approaches) of new individuals for mental domains and in $73.5 \%(S D=1.9 \%)$ for experimental tasks on average. Predictive models aware of interindividual differences in brain structure outperformed predictive models based on functional and combined imaging modalities in four of nine brain sampling approaches in mental domains and in five of nine approaches in experimental tasks. We further observed that the average classification accuracy of predictive models of meta-priors informed by brain function reached $70.9 \%(S D=1.6 \%)$ for mental domains and $70.8 \%$ $(S D=1.4 \%)$ for experimental tasks. The predictive models that only had access to brain function were found to be superior to structural and combined imaging modalities in 1 of 9 brain sampling approaches in mental domains and in zero of nine brain sampling approaches in experimental tasks. In combined brain structure and function, we found mean classification performances of $73.4 \%(S D=1.9 \%)$ in mental domains and $73.6 \%(S D=2.1 \%)$ in experimental tasks. The predictive model tuned to combined information from brain structure and brain function performed better than predictive models based on a single imaging modality in some but not all brain sampling approaches in both mental domains and experimental tasks. Across different types of brain information, these slight differences in classification performance were not statistically significant at $p<.05$ as indicated by our bootstrapped $95 \%$ confidence intervals. On average, however, predictive models utilizing both brain structure and brain function achieved the highest classification performances.

We then examined the outcome of different brain sampling approaches (Figure S2). We observed varying prospective classification performances depending on the type of brain data. In combined brain structure and function, for instance, the classification performance ranged from 69.8\% ([57.6\%; 81.3\%], bootstrapped 95\% $\mathrm{Cl}$ ) to $75.9 \%$ ([62.5\%; 84.4\%]) across mental domains. Among experimental tasks, in turn, the prediction accuracy ranged from 69.8\% ([60.6\%; $81.3 \%])$ to $75.9 \%$ ([68.8\%; $87.9 \%]$ across different data-extraction approaches. Comparing the different brain sampling approaches, different activation-, region-, or network-focused strategies were 
advantageous in different settings for separating patients from controls, without a consistent winner.

It is important to note that our analyses were based on brain data after adjusting for age, sex, and data acquisition site to prevent the predictive model from capturing variation due to variables of no interest. In brain data without this confound removal step, we observed instances of slightly elevated classification performance of the predictive model. This piece of evidence suggests that the predictive model learned useful information from nuisance variables. Nevertheless, the uncorrected subanalyses yielded a comparable ranking order, which provided evidence that the relevant brain variation was mostly independent of age, sex, and site.

\subsection{Testing the cognitive specificity of schizophrenia predictability}

Next, we performed a negative test to further ensure that the predictive model captured the individual constellation of cognitive domains instead of potential confounding influences. We formally compared the classification performance of the domain-spanning model against a cognition-naïve null model. In 1,000 random permutations, we specifically permuted how domain combinations co-occurred within patients with schizophrenia or healthy controls. By preserving all other data characteristics, we tested whether the constellation of cognitive functions of the participants were relevant for group classification. The nonparametric hypothesis test revealed that the summary model discriminated between patients and controls significantly better than the null model across brain sampling approaches at a level of $p<.05$ for mental domains and $p<.01$ for experimental tasks (Figure 3). That is, we observed our actual or a higher prediction accuracy in less than 50 of 1,000 cases in mental domains and in less than 10 in 1,000 cases in experimental tasks if there was no systematic relation between an individual's cognitive relevance and group detection. In short, our negative test ascertained that the successful classification performance of the composite predictive model could be defensibly ascribed to the participant-specific configurations of cognitive aspects.

\subsection{Determining contributions to schizophrenia predictability across cognitive domains}

The domain-spanning summary model enabled the direct comparability of the cognitive meta-priors despite their naturally varying cortical and subcortical spread. That is, the taxonomy-level predictive model enabled us to impartially contrast the cognitive processes in their relative contribution to schizophrenia classification.

Informed by both brain structure and function, the rankings of mental domains (Figure 4) and experimental tasks (Figure 5) clearly demonstrated that some candidate processes were often more relevant for schizophrenia detection than others. Regarding mental domains, for instance, the top-scoring domains of gustatory perception, pain perception, and experience of sadness were found to be statistically significantly more predictive of schizophrenia than

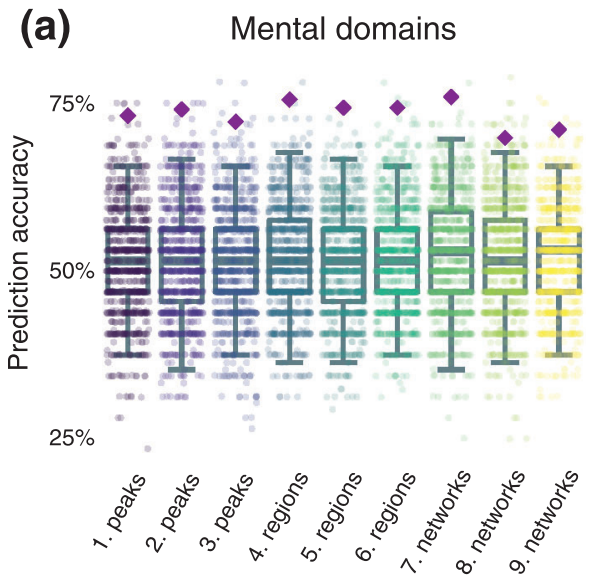

Pipelines (b) Experimental tasks

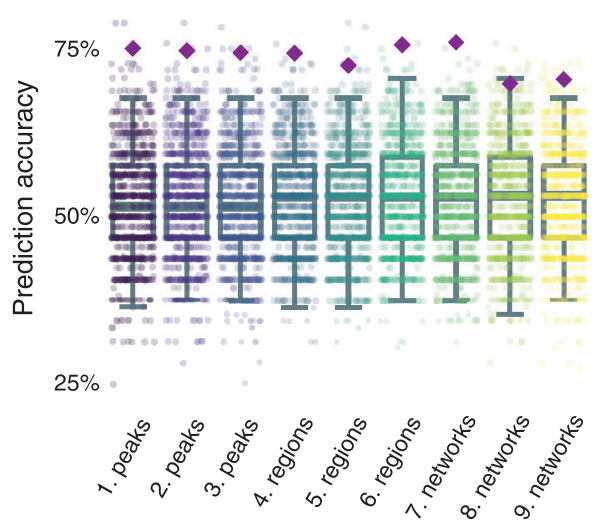

Pipelines

FIGURE 3 Validation of our data-analysis framework. The final predictive model classified healthy versus schizophrenic individuals statistically significantly better than a cognition-naive null model in each of two taxonomies. We estimated the null distribution by selectively corrupting the participant pattern of cognitive indices while leaving other structure in the data intact. To ascertain that the final predictive model captured participant-specific cognitive facets instead of confounding variables. Purple diamonds indicate the (out-of-sample) classification performance of the composite model based on (a) mental domains and (b) experimental tasks using combined structural (sMRI) and functional (fMRI) brain information. The dots show 1,000 model performances realized under the null hypothesis. The gray boxplots show bold lines for median (50th percentile), the lower and upper quartile (25th and 75th percentiles), and whiskers for the interquartile distance (25th-75th percentiles) besides the box. In each of nine ways to sample brain information, the composite model performed significantly better than the null model ( $p<.05$ for mental domains and $p<.01$ for experimental tasks). If the individual combinations of cognitive expressions were not relevant, we would only observe our actually obtained prediction performance (purple diamond) in at most 50 out of 1,000 cases for mental domains and in at most 10 out of 1,000 cases for experimental tasks. The negative test implies that the successful individualized decisions of our predictive model can be ascribed to participant-specific cognitive alterations rather than other characteristics of the participant sample [Color figure can be viewed at wileyonlinelibrary.com] 


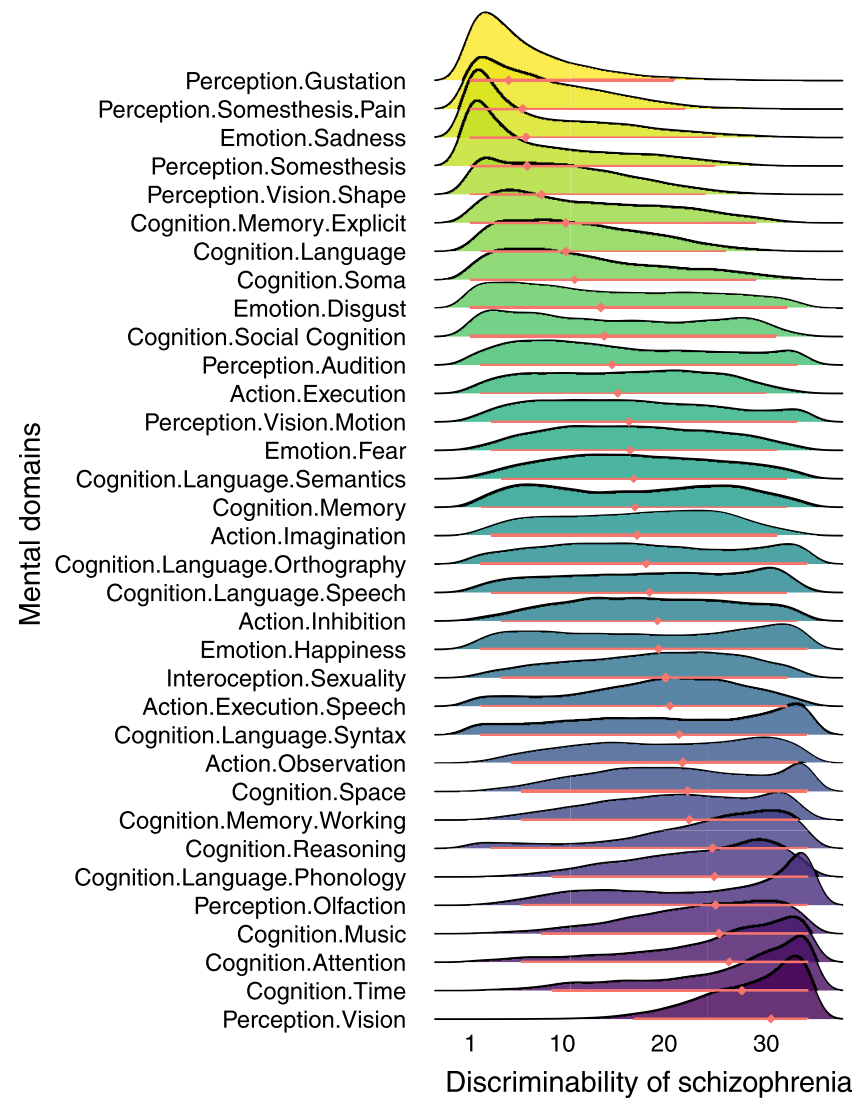

FIGURE 4 Quantified predictive value of mental domains in schizophrenia (SCZ). We systematically screened for dysregulated cognitive processes to facilitate the development of personalized diagnoses and new treatment strategies. Relative contribution of mental domains in disambiguating patients with $\mathrm{SCZ}$ and healthy controls. Thirty-four mental domains ordered according to their average ability to forecast disease status. Joyplot shows weighted importance ranks for each domain (colored mountains). Red diamonds depict mean ranking position across brain sampling strategies (see Figure S3 for pipeline-specific domain ranks). Certainty of discriminability position was assessed by estimating bootstrapped 95\% population intervals (red lines). For instance, gustation was highly predictive across complementary approaches to sample neurobiological information, whereas the relevance of audition was more dependent on the sampling pipeline. Some intensively studied concepts of attention (e.g., Braff, 1993) and working memory (e.g., Forbes et al., 2009; Lee \& Park, 2005) have been situated among the cognitive classes least predictive for SCZ. All results based on combined SMRI and fMRI data [Color figure can be viewed at wileyonlinelibrary.com]

attention, temporal reasoning, and visual perception at $p<.05$ (Figure 4). Regarding experimental tasks, mental processes related to pain discrimination, face discrimination, and visual tracking were significantly more discriminable of disease status than the lowest ranked mental operations underlying listening to and producing music, visuospatial attention, and the Stroop task at a level of $p<.05$ (Figure 5). Across both taxonomies, we observed that cognitive functions related specifically to social affective (e.g., experience of sadness and face discrimination tasks) and internally oriented perception processes (e.g., pain perception and

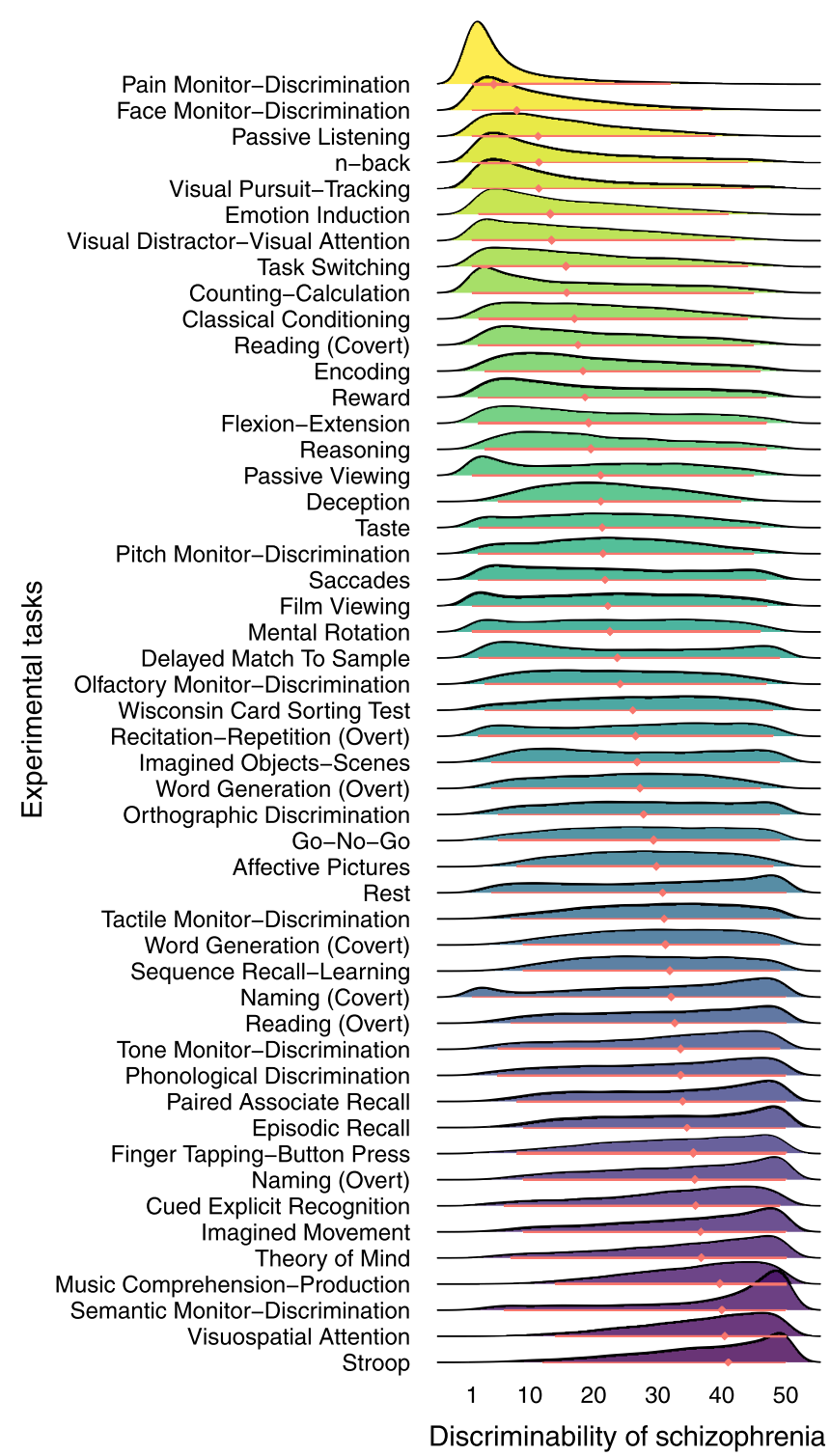

FIGURE 5 Quantified predictive value of experimental tasks in schizophrenia (SCZ). We broadly screened for distinctive experimental paradigms to facilitate the development of personalized diagnoses and new treatment strategies. Relative contribution of experimental tasks in disambiguating patients with SCZ and healthy controls. Fifty experimental tasks were ordered according to their average ability to forecast disease status across brain sampling strategies (see Figure 4 for pipeline-specific domain ranks). Joyplot shows weighted importance ranks for each domain (colored mountains). Red diamonds depict mean position in relevance. Certainty of discriminability position was assessed by estimating $95 \%$ population intervals (red lines). Precision estimates computed by repeatedly resampling participants with replacement (bootstrapping). All results based on combined sMRI and fMRI data [Color figure can be viewed at wileyonlinelibrary.com]

pain discrimination tasks) emerged as most critical for schizophrenia classification. Thus, we quantitatively identified common and distinct elements of cognition in MRI-based imaging of brain structure and function in their utility for the study of schizophrenia. 
We further elaborated this evidence from combined brain structure and function in predictive models that drew on a single imaging modality. In brain volume alone, 7 of the top 10 mental domains were in agreement with the top 10 mental domains found in combined SMRI and fMRI data. However, knowledge of speech, sensing sexual needs, and speaking were ranked among the top 10 mental domains in brain structure instead of pain perception, knowledge of language, and body knowledge. Similarly, 5 of the top 10 ranked experimental tasks in brain structure were also counted among the top 10 experimental tasks in combined brain structure and function. Yet, mental processes elicited by film viewing, encoding, theory of mind, passive viewing, and Wisconsin Card Sorting Test tasks emerged as more disease relevant in brain structure than mental operations involved in visual tracking, visual attention, task switching, numerical operations, and classical conditioning experiments. In intrinsic functional connectivity alone, 6 of the top 10 mental domains corresponded to the top 10 mental domains in combined sMRI and fMRI data. However, motor execution, motion perception, experience of fear, and auditory perception were counted among the top 10 mental domains in brain function instead of knowledge of language, body knowledge, experience of disgust, and social cognition. Similarly, 7 of the top 10 ranked experimental tasks in brain function were concordant with the top 10 found in combined brain structure and function. Here, mental operations related to flexing and extending movements, rapid eye movements, and passive viewing tasks arose as more discriminable of schizophrenia in brain function than mental processes elicited by face discrimination, task switching, and numerical operations paradigms. Despite several modality-specific relevances of cognitive domains in schizophrenia, the obtained utility rankings were largely overlapping based on different types of brain data.

\section{5 | Isolating nonlinear predictive relationships across cognitive domains and predictive relevance maps}

Finally, we wished to explore how cognitive processes act in more complicated ways together in distinguishing between patients and controls (Figure 6). For this purpose, we estimated the nonlinear interaction of cognitive domain pairs in schizophrenia prediction. The so-called "partial dependence" estimation quantified the relationship between two cognitive meta-priors in predicting disease status, after accounting for the effects of the remaining domains of the taxonomy. In other words, we investigated the joint contribution of two cognitive processes in schizophrenia prediction while accommodating the influence of the remaining domains. Charting these two-way interactions of the most predictive mental domains and experimental tasks showed various types of links between schizophrenia classification and single cognitive concepts. Besides approximately linear links to schizophrenia prediction (n-back task and passive listening), we also observed somewhat logarithmic (experience of sadness), exponential (pain perception and pain discrimination), and polynomial (gustatory perception and face discrimination) nonlinear relationships. Similarly, we found different qualities of relationships such as of approximately linear (semantic discrimination and Stroop task), logarithmic (visual perception), exponential (temporal reasoning), and polynomial kind (listening to and producing music) among the lowest ranked domains (Figure S5). The comparison of the more-than-linear effects of two top ranked and two less successful domains also showed that schizophrenia classification relied more heavily on statistical dependencies among the top ranked domains as compared to the lowest ranked domains. When we directly contrasted top and lowest ranked domains, we again found stronger contributions of the top ranked domains on the classification of health versus disease compared to the lowest ranked domains (e.g., gustatory perception vs. attention, and face discrimination vs. Stroop task). Across imaging modalities and cognitive description catalogs, we observed that cognitive functions contributed in complex ways to schizophrenia classification, that is, patterns in brain data to which purely linear Pearson correlation and regression-type analyses are blind.

After contrasting the individual meta-priors for their differences, we wished to explore common characteristics across the cognitive classes of a taxonomy in schizophrenia prediction. To examine which brain areas were most discriminative of disease status across cognitive domains, we globally mapped the importance of each cognitive metaprior onto the brain. In both mental domains and experimental tasks, we observed largely overlapping patterns of brain regions that were most relevant for disease classification (Figure 7). This similarity across two distinct ways to catalog cognitive functions serves as post hoc validation of our approach.

\section{4 | DISCUSSION}

How can we derive principled recommendations for psychology and neuroscience experiments from brain recordings that can be measured at scale? We have introduced a machine-learning strategy to stratify a catalog of cognitive classes according to their utility in identifying schizophrenia. We first distilled existing neurobiological knowledge on constituent elements of the human mind into couples of cognitive concept and quintessential neural representation. In a data-driven fashion, these cognitive meta-priors were contrasted in their capacity to distinguish between patients and controls based on easily acquired and commonly available structural and functional brain scans of a multisite study of a large schizophrenia cohort. Each domain-specific classifier was exclusively based on brain information with a pre-established link to a specific cognitive domain. The analytical framework was impartial in giving each cognitive category the same opportunity to be selected as most important in identifying schizophrenia. The data-guided ranking highlighted certain cognitive categories that were more discriminative for this major psychiatric disorder than other types of mental activity. It is key outcome that we found both frequently investigated and largely untapped disease concepts to be relevant in schizophrenia.

Among the traditionally examined concepts, our across-systems analysis underscored the critical role of tones and speech appraisal (passive listening) as third most relevant among 50 experimental tasks and the sense of hearing (audition) as 11th among 34 mental domains, on the one hand. This quantitative evidence from structural and functional brain scans confirms the long-standing clinical emphasis on 
(a)
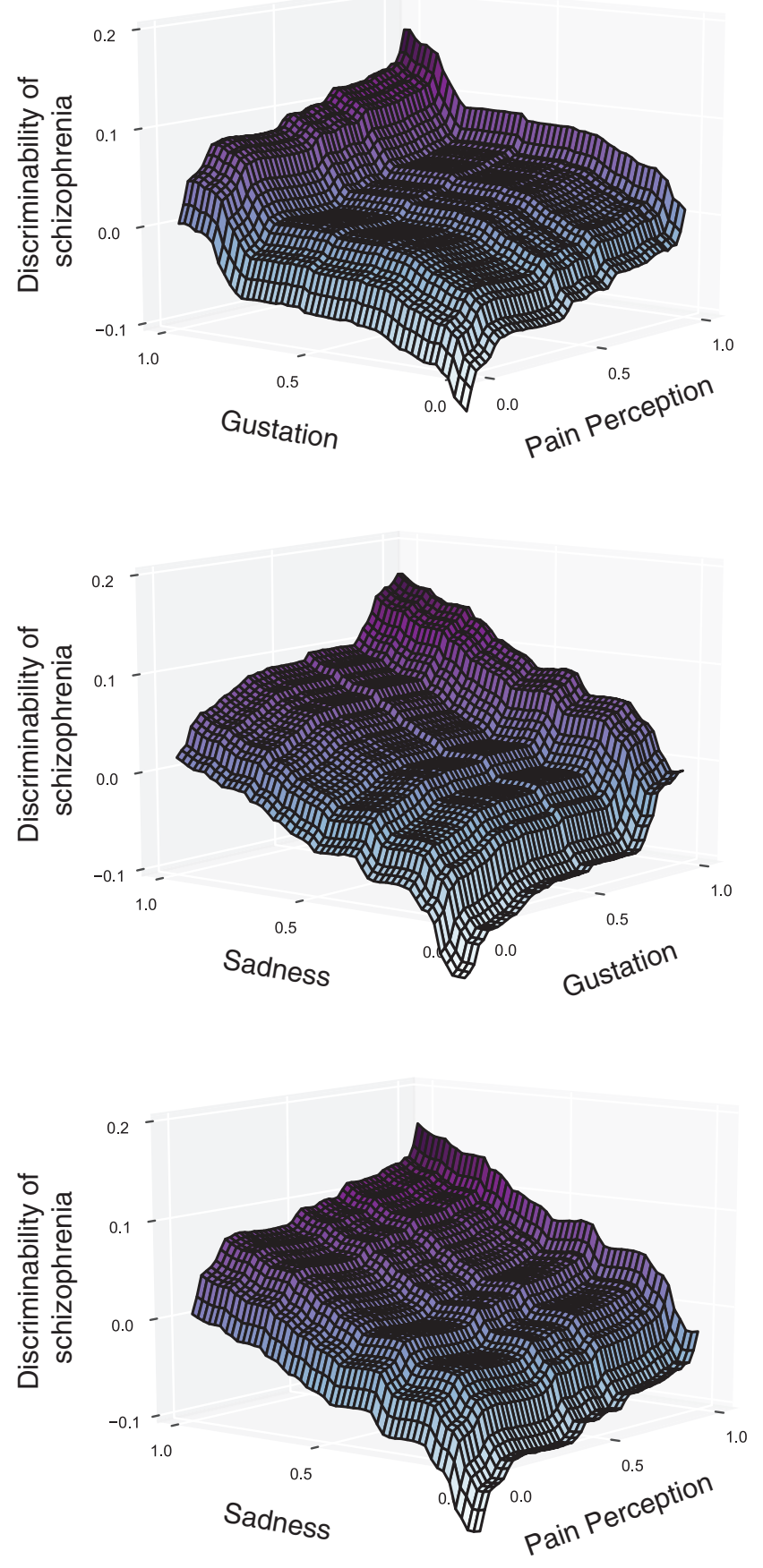

(b) Experimental tasks
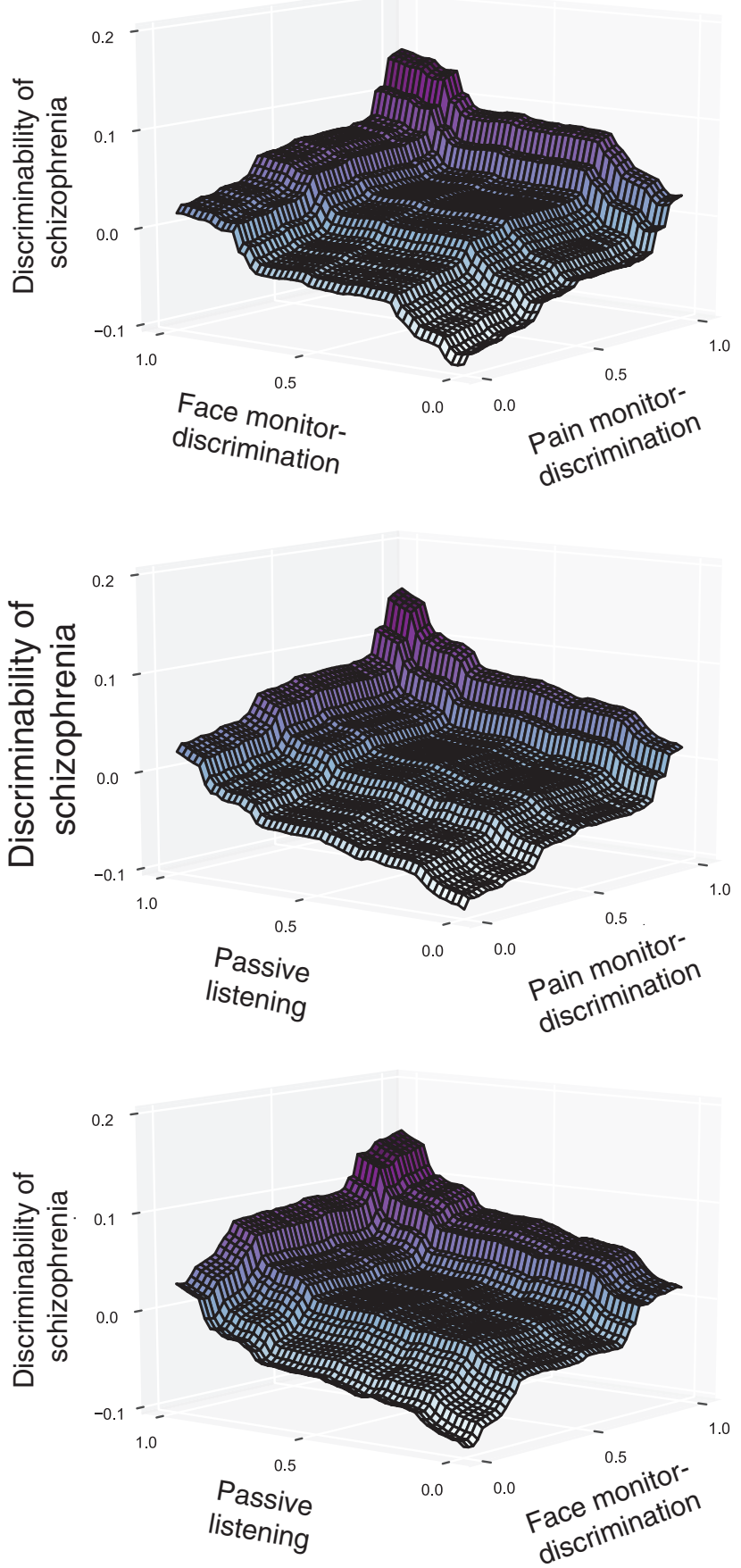

FIGURE 6 Domain-domain interactions in detecting schizophrenia (SCZ). Inspects in more detail potentially complicated relationships in the predictability of (a) mental domains and (b) experimental tasks after accounting for the influence of the remaining domains (instead of ignoring them). Partial dependence of SCZ predictability ( $z$-axis) on the joint distribution of two selected cognitive domains ( $x$ - and $y$-axis) in predicting whose brain scans are from a SCZ patient (Principal component analysis [PCA] pipeline). Pairs of the top three cognitive processes are shown for both taxonomies (see Figure S5 for further examples). All results based on combined brain imaging types (sMRI and fMRI data) [Color figure can be viewed at wileyonlinelibrary.com]

auditory hallucinations as a hallmark symptom of schizophrenia patients. Hearing malevolent voices and other auditory misperceptions (Lim, Hoek, Deen, \& Blom, 2016; Llorca et al., 2016; McCarthyJones et al., 2017) might be mediated by impaired preattentive filtering mechanisms (Javitt, 2009; Javitt \& Freedman, 2015; Javitt \&
Sweet, 2015; Rissling \& Light, 2010). Additionally, neuroimaging studies have consistently shown abnormalities of auditory brain regions in schizophrenia using meta-analyses: (a) structural neuroimaging studies found reduced volume in parts of the auditory cortex in the superior temporal gyrus (Honea, Crow, Passingham, \& Mackay, 2005; Modinos 

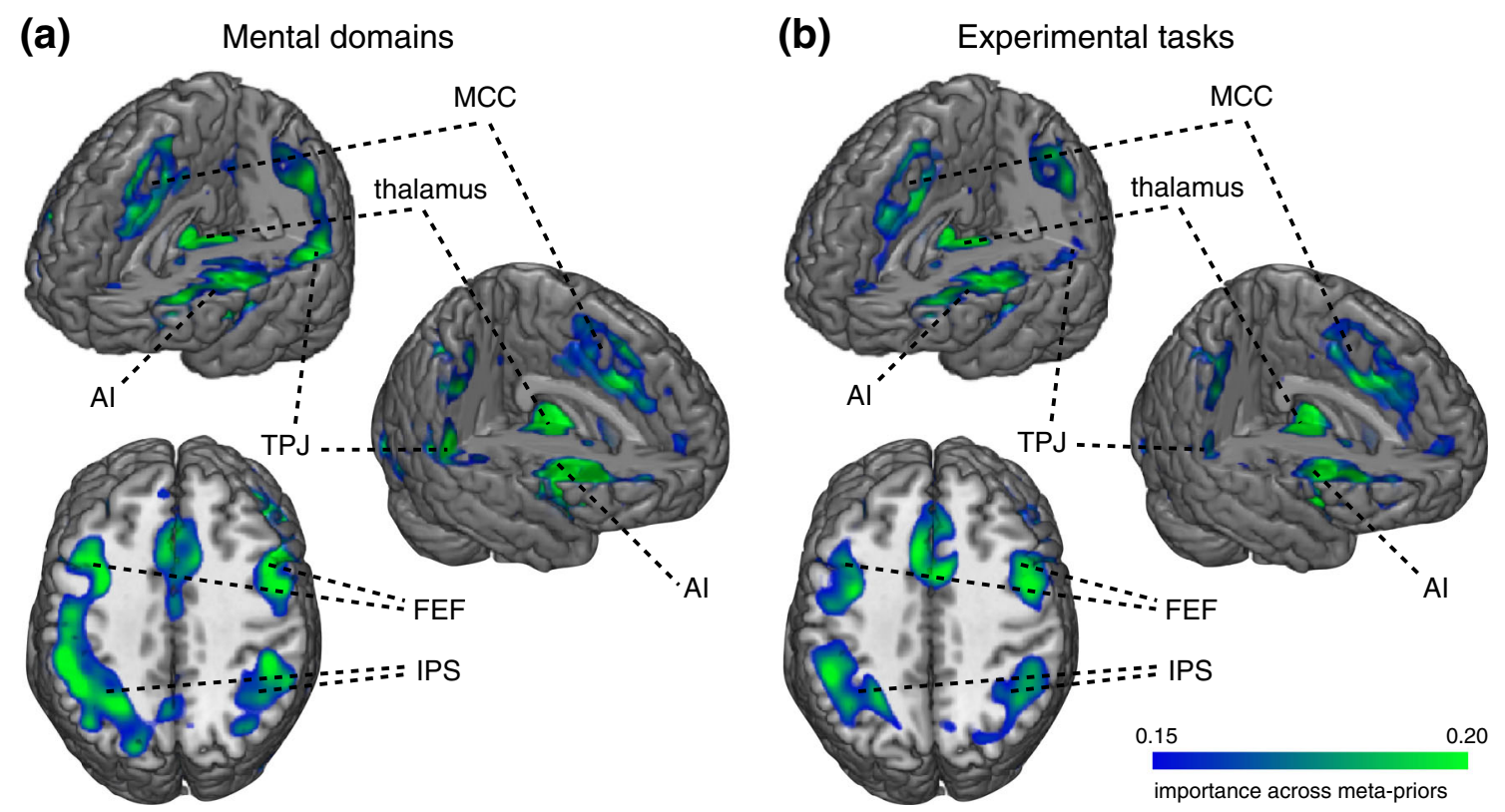

FIGURE 7 Predictive relevance maps for schizophrenia (SCZ). Quantifies the average extent to which individual brain regions contributed to disease classification across (a) 34 mental domains and (b) 50 experimental tasks. Whole-brain maps depict relative importance values of the cognitive meta-priors across nine brain sampling strategies. In both taxonomies, nodes of the dorsal attention network (e.g., frontal eye field [FEF] and intraparietal sulcus [IPS]) and saliency network (e.g., anterior insula [AI] but not mid cingulate cortex [MCC]) as well as thalamus were highly pertinent in distinguishing patients from controls. Left and right temporo-parietal junction (TPJ), however, emerged as discriminative in mental domains but not in experimental tasks. Both taxonomies provided largely overlapping but still distinct brain patterns underlying SCZ classification. This converging evidence across two independent cognitive taxonomies further strengthens the validity of our approach. Brain maps were smoothed (FWHM $=6 \mathrm{mmm}$ ) and thresholded for display (see https://neurovault.org/collections/4074/ for unthresholded predictive brain maps). All results are based on combined SMRI and fMRI data [Color figure can be viewed at wileyonlinelibrary.com]

et al., 2013) and (b) functional investigations have reported increased neural activity in areas related to speech perception, language, and memory during active sensations of speech hallucinations (Allen et al., 2012; Jardri, Pouchet, Pins, \& Thomas, 2011).

A relation of auditory processing systems with language and memory was suggested by theories that ascribe the formation of auditory hallucinations to intrusive memories and the external misattribution of inner speech (Curcic-Blake et al., 2017; Jardri et al., 2011). In line with these notions, our stratified prediction experiment revealed the relevance of memory and language systems (explicit memory and language). Besides the auditory system, also visual processing (e.g., shape vision and visual tracking) emerged as disease-relevant-a sense commonly affected by hallucinations and psychosis (Waters et al., 2014). The disturbance of these basic sensory processes might lead to deficits in higher level cognition and thus impact functional outcome in patients, which is supported by a recent structural equation modeling study (Javitt \& Freedman, 2015; Thomas et al., 2017). Difficulties in the interpretation of speech prosody were argued to potentially entail problems in social interaction (Javitt \& Freedman, 2015; Javitt \& Sweet, 2015). Our large-scale analyses provide evidence for the potential of MRI-facilitated clinical control especially for distressing and at times dangerous voice hallucinations.

Furthermore, viewing or evaluating information from others' faces (face discrimination) as a core element of social cognition was attributed second highest importance among 50 experimental tasks pooling across brain structure and function. Concurrently, emotional processing, as another important aspect of social behavior (Green, Horan, \& Lee, 2015; Ochsner, 2008), emerged as relevant for schizophrenia: appraisal of environmental cues with affective valence (emotion induction) was ranked sixth among 50 experimental tasks, while the experience of two negative basic emotions, sadness, and disgust, were ranked third and ninth among 34 mental domains. The more general concept of information processing related to fellow humans (social cognition), was 10th among 34 mental domains. By carrying out a quantitative cognitive screening, we endorse the broader relevance of social-affective thought and behavior in schizophrenia. This comparably recent research trend in psychiatry and neuroscience is currently gaining momentum for several reasons: (a) previous studies have hinted at a broad spectrum of social dysfunctions in patients with schizophrenia (see Savla, Vella, Armstrong, Penn, and Twamley (2013) for a meta-analysis), (b) such dysfunctions seem to be timeenduring and already present in prodromal phases of the disease (Bora, Yucel, \& Pantelis, 2009; Green et al., 2012), and (c) the deficits in social cognition are intimately related to poor work and community functioning (Couture, Penn, \& Roberts, 2006; Fett et al., 2011; McCleery et al., 2016). Moreover, aberrations in socio-emotional processing circuits seem to mediate the impact of social environmental risk factors such as urbanization and migration on schizophrenia (Tost \& Meyer-Lindenberg, 2012).

Our quantitative outcomes are also supported by earlier documentation of emotional processing disturbances in schizophrenia (Aleman \& Kahn, 2005; Derntl et al., 2009; Derntl et al., 2012). Several emotion recognition studies reported larger impairments in the processing of faces with negative, rather than positive, emotions such 
as sadness and disgust (Kohler et al., 2010). The predominance of impaired negative emotion recognition was also reflected in our results by higher disease discriminability of negative emotions such as sadness, disgust, and fear as opposed to the positive emotion happiness. In line with our evidence, a meta-analysis of structural MRI studies in schizophrenia highlighted gray matter decreases in amygdala and insula (Ellison-Wright, Glahn, Laird, Thelen, \& Bullmore, 2008). Another MRI study found a positive correlation between amygdala volume and performance in recognizing sad faces (Namiki et al., 2007). Furthermore, a meta-analysis of functional MRI studies revealed reduced brain activation in amygdala, parahippocampal gyrus, and fusiform gyrus, but increased activity in left insula during emotional face processing (Li et al., 2010).

The aberration of social-affective brain systems in schizophrenia also translates into various clinical symptoms. Patients often exhibit negative symptoms, such as diminished emotional expression and apathy, which tend to have enduring trajectories compared to the more episodic positive symptoms. Indeed, impairments in social cognition have been proposed to have a stronger impact on functional outcome than other cognitive impairments (Carrión et al., 2016; Fett et al., 2011). The collective outcomes of our cognitive charting underscore the potential of clinical interventions that target affective impairments in schizophrenia such as social rehabilitation and training regimens in various social skills (Kurtz \& Richardson, 2012).

Besides reinforcing currently studied forms of thinking aberration, certain cognitive domains emerged as critical to schizophrenia patients that have so far seldom been the center of investigation. For instance, the appraisal of pain-related cues was ranked as the first most predictive experimental task (pain discrimination) and second most affected mental domain (pain perception). Despite a documented decrease in the sensitivity of patients to pain stimuli in the clinical setting, this phenomenon has been the object of very few experimental studies (Dworkin, 1994; Stubbs et al., 2015). A recent review on the limited available literature suggested the existence of impairments in the sensory-discriminative, affective, and cognitive components of pain processing (Stubbs et al., 2015). Similarly, one of the few existing neuroimaging studies on the topic reported decreased recruitment of pain-responsive brain regions such as the anterior insula and increased recruitment of sensory-processing-tuned brain systems such as the primary somatosensory cortex during pain processing in patients with schizophrenia (de la Fuente-Sandoval, Favila, Gomez-Martin, Pellicer, \& Graff-Guerrero, 2010).

Combining these streams of evidence, we used our cognitive ranking outcomes to identify a critical role of pain and emotion appraisal in schizophrenia. In combination with the highly scored domains related to processing external and internal bodily feedback such as skin sensations (somatic cognition and somesthesis), many of the most predictive cognitive classes can be considered as impaired interoceptive integration. This contention of a key role of binding bodily information is further supported by the first rank of the sense of tasting (gustation). Indeed, misperceptions of imagined tactile or bodily cues (e.g., feeling of insects on the skin) are an often-encountered clinical symptom in schizophrenia patients (McCarthy-Jones et al., 2017;
Thomas et al., 2007). More broadly, this major psychiatric disease is often considered to be a disorder of the self and subjective experience (Fletcher \& Frith, 2009). Colloquially, these patients may suffer from a misbalance in "how the body listens to itself"-how it senses, integrates, and prepares reactions to somatic signals. Alterations of interoceptive capacity might also give rise to misattribution of inner signals to external sources, which is a frequently observed clinical feature of patients with schizophrenia. Dysregulated processing of nociceptive and autonomic signals has very recently been raised as a potential mechanism involved in schizophrenia pathophysiology and potentially other mental disorders (Ardizzi et al., 2016; Khalsa et al., 2017; Owens, Allen, Ondobaka, \& Friston, 2018). More generally, pain insensitivity can lead to poor help-seeking behavior. Hence, future research endeavors enlightening pain processing in schizophrenia might help to reduce the high morbidity and mortality observed among schizophrenia patients (Dworkin, 1994; Stubbs et al., 2014; Stubbs et al., 2015). Both pain appraisal and gustation are exemplary instances of the used taxonomy that shares the processing of internal bodily information and associated feedback loops. Taken together, the identification of these highly discriminative cognitive classes stresses the research potential of elucidating how bodily signals from the internal organs which may expose a currently underappreciated disease mechanism.

This interpretation is in line with another quantitative finding of a yet mostly unexplored domain. Tasting or imagining the flavor of food reached the first position among 34 candidate mental domains which probably relates to epidemiological studies that estimate $7-31 \%$ of patients with schizophrenia experience some form of gustatory hallucination (Baethge et al., 2005; Connolly \& Gittleson, 1971; Lewandowski, DePaola, Camsari, Cohen, \& Ongur, 2009; Thomas et al., 2007). The scarce studies directly examining gustation in patients with schizophrenia reported a significant deficit in their sensitivity for different tastes (Balderston et al., 2003), such as the bitter-tasting antiheroic compound phenylthiocarbamide (Moberg et al., 2005; Moberg et al., 2007). Additionally, there is some tentative evidence for abnormalities in brain regions related to gustation including the insula, thalamus, and orbitofrontal cortex (Balderston et al., 2003). For instance, taste chemoreceptors responses seem to be reduced in the dorsolateral prefrontal cortex (Ansoleaga et al., 2015). The subordinate role of gustatory hallucinations in common clinical assessments, as opposed to other sensory misperceptions, may be one reason why research on disturbed gustatory processing in schizophrenia may still be in its infancy.

While our study capitalized on the BrainMap database, alternative coordinate-based databases hosting task fMRI results need to be mentioned. As a key representative, Neurosynth hosts a large amount of task fMRI studies with their foci of significant peak neural activity changes (neurosynth.org). In stark contrast to BrainMap, Neurosynth labels the neuroimaging results in a bottom-up fashion by searching for cognitive terms mentioned by the authors themselves throughout their scientific papers. This automated text-mining strategy has the important advantage to extract annotations of published neuroimaging experiments in a fast and efficient way. This allows a natural scaling to collect always more neuroimaging publications. However, NeuroSynth's approach yields drawbacks for our specific goal to 
systematically test diverse cognitive functions for their relevance in schizophrenia. First, a given cognitive term can refer to different cognitive processes (e.g., "speech" can refer to both the motor action of speaking and the semantic processing underlying language production as explicitly distinguished by the BrainMap taxonomy). Second, different commonly used cognitive terms can denote identical or similar cognitive processes due to the lack of a consensus definition among neuroscientists (e.g., "emotional," "affective," and "motivational"). Third, the authors mentioning a cognitive term somewhere in the paper text does not guarantee that this cognitive process was directly examined in the experiment, or that it is necessarily mentioned within the context of the conducted experimental contrast whose coordinates are extracted. To alleviate some of these inadequacies, BrainMap has cataloged each experiment in accordance with two expert-developed and continuously refined cognitive description systems. In addition, all taxonomy assignments were verified by different scientists to strive toward a clean and consistent annotation of the neuroimaging experiments with the cognitive categories. As such, the BrainMap database and annotation principle was more suitable for the question at the heart of our present investigation.

While we provide valuable meta-level insights into what cognitive processes might be most dysfunctional in schizophrenia, our computational modeling approach reached somewhat lower performance in disease classification than certain previous machine-learning studies that were solely focused on prediction performance alone (e.g., Silva et al., 2014). This observation was entirely expected. In statistical data analysis in general, there is a widely recognized tension between predictive performance and model interpretability (Bishop, 2006; Danilo Bzdok \& Yeo, 2017; Hastie et al., 2001). Machine-learning algorithms are particularly suited to achieve highly accurate predictions in a brute-force fashion, which is why they might be promising for precision psychiatry (Danilo Bzdok \& Meyer-Lindenberg, 2018; Chekroud, Lane, \& Ross, 2017). However, such purely data-driven approaches were sometimes criticized for offering less direct insight into the cognitive or neurobiological architecture of schizophrenia. Acknowledging the often-incompatible goals of revealing the underpinnings of a disease and best-possible bare prediction outcomes, our study prioritized the interpretability of the statistical framework. The introduction of condensed neuroscientific knowledge in a principled fashion enabled the study of compromised cognitive processes in a major psychiatric disease. The compression of the original brain information into interpretable summaries came at the expense of best-possible prediction accuracy (Hastie et al., 2001; Kuhn \& Johnson, 2013; Shmueli, 2010). Additionally, the classification performance of our predictive model might be compromised by using a multisite dataset. Such samples often introduce additional sources of variance that differ across sites (e.g., scanner software) (Dansereau et al., 2017; Nielsen et al., 2013). Nevertheless, clinical samples with patients from several sites are often more representative of the general population and are hence more likely to provide clinically relevant and reproducible results (Abraham et al., 2017; Costafreda, Brammer, David, \& Fu, 2008).

A further strength of our computational neurocognitive assay lies in leveraging accumulated neurobiological knowledge of executed behaviors and their recruited neural systems to build a single-patient prediction framework (Arbabshirani, Plis, Sui, \& Calhoun, 2017; Huys, Maia, \& Frank, 2016; Stephan et al., 2017). Such integration of data-driven and theoryassisted analysis tactics allowed us to discover the most impaired cognitive domains in a rich schizophrenia sample while relying on minimal additional statistical, pathophysiological, and neurobiological assumptions. In this way, our study exemplified hypothesis mining on which components of human cognition might be particularly affected in a particular psychiatric disorder. These most promising candidate neural systems can provide a well-founded basis for explicit hypotheses testing on multiple levels of observations, such as genomics, neurotransmitters, and neuropharmacology. We are optimistic that such computational psychiatry investigations could be readily extended to many brain disorders and potentially inform priority agendas in health research (Oquendo et al., 2012).

\section{ACKNOWLEDGMENTS}

T.M.K. is funded by a full $\mathrm{PhD}$ scholarship of the German National Merit Foundation. Dr. D.B. is funded by the Deutsche Forschungsgemeinschaft (DFG, BZ2/2-1, BZ2/3-1, and BZ2/4-1; International Research Training Group IRTG2150), Amazon AWS Research Grant (2016 and 2017), as well as the START-Program of the Faculty of Medicine (126/16) and Exploratory Research Space (OPSF449), RWTH Aachen. Dr. D.S.B. acknowledges support from the ISI Foundation, the Alfred P. Sloan Foundation, the John D. and Catherine T. MacArthur Foundation, and the Paul Allen Foundation.

\section{CONFLICT OF INTEREST}

The authors declare no competing conflict of interest.

\section{DATA AVAILABILITY}

The neuroimaging results that have been used to generate the cognitive meta-priors are openly available in the BrainMap database (http://www.brainmap.org/).

\section{ORCID}

Teresa M. Karrer (D) https://orcid.org/0000-0002-7444-7679 Danielle S. Bassett (D) https://orcid.org/0000-0002-6183-4493 Bertrand Thirion (iD https://orcid.org/0000-0001-5018-7895

\section{REFERENCES}

Abraham, A., Milham, M. P., Di Martino, A., Craddock, R. C., Samaras, D., Thirion, B., \& Varoquaux, G. (2017). Deriving reproducible biomarkers from multi-site resting-state data: An autism-based example. Neurolmage, 147, 736-745. https://doi.org/10.1016/j.neuroimage. 2016.10.045

Abraham, A., Pedregosa, F., Eickenberg, M., Gervais, P., Mueller, A., Kossaifi, J., ... Varoquaux, G. (2014). Machine learning for neuroimaging with scikitlearn. Frontiers in Neuroinformatics, 8(14), 1-10. https://doi.org/10.3389/ fninf.2014.00014 
Aleman, A., \& Kahn, R. S. (2005). Strange feelings: Do amygdala abnormalities dysregulate the emotional brain in schizophrenia? Progress in Neurobiology, 77(5), 283-298. https://doi.org/10.1016/j.pneurobio.2005. 11.005

Allen, P., Modinos, G., Hubl, D., Shields, G., Cachia, A., Jardri, R., ... Hoffman, R. (2012). Neuroimaging auditory hallucinations in schizophrenia: From neuroanatomy to neurochemistry and beyond. Schizophrenia Bulletin, 38(4), 695-703. https://doi.org/10.1093/schbul/sbs066

Ansoleaga, B., Garcia-Esparcia, P., Pinacho, R., Haro, J. M., Ramos, B., \& Ferrer, I. (2015). Decrease in olfactory and taste receptor expression in the dorsolateral prefrontal cortex in chronic schizophrenia. Journal of Psychiatric Research, 60, 109-116. https://doi.org/10.1016/j. jpsychires.2014.09.012

Arbabshirani, M. R., Plis, S., Sui, J., \& Calhoun, V. D. (2017). Single subject prediction of brain disorders in neuroimaging: Promises and pitfalls. Neurolmage, 145, 137-165.

Ardizzi, M., Ambrosecchia, M., Buratta, L., Ferri, F., Peciccia, M., Donnari, S., ... Gallese, V. (2016). Interoception and positive symptoms in Schizophrenia. Frontiers in Human Neuroscience, 10(379), 1-10. https://doi.org/10.3389/fnhum.2016.00379

Ashburner, J., \& Friston, K. J. (2005). Unified segmentation. Neurolmage, 26(3), 839-851.

Baethge, C., Baldessarini, R. J., Freudenthal, K., Streeruwitz, A., Bauer, M., \& Bschor, T. (2005). Hallucinations in bipolar disorder: Characteristics and comparison to unipolar depression and schizophrenia. Bipolar Disorders, 7(2), 136-145. https://doi.org/10.1111/j.13995618.2004.00175.x

Balderston, K., Moberg, P., Turetsky, B., Palecanda, B., Doty, R., \& Gur, R. (2003). Deficits in regional taste sensitivity in patients with schizophrenia. Schizophrenia Research, 60(1), 122.

Bishop, C. M. (2006). Pattern recognition and machine learning. New York, NY: Springer.

Bora, E., Yucel, M., \& Pantelis, C. (2009). Theory of mind impairment in schizophrenia: Meta-analysis. Schizophrenia Research, 109(1-3), 1-9. https://doi.org/10.1016/j.schres.2008.12.020

Bossier, H., Seurinck, R., Kühn, S., Banaschewski, T., Barker, G. J., Bokde, A. L. W., ... Moerkerke, B. (2018). The influence of study-level inference models and study set size on coordinate-based fMRI metaanalyses. Frontiers in Neuroscience, 11(745) 1-22. https://doi.org/10. 3389/fnins.2017.00745

Braff, D. L. (1993). Information processing and attention dysfunctions in schizophrenia. Schizophrenia Bulletin, 19(2), 233-259.

Breiman, L. (1996). Stacked regressions. Machine Learning, 24(1), 49-64. https://doi.org/10.1007/bf00117832

Breiman, L. (2001). Random forests. Machine Learning, 45(1), 5-32.

Bzdok, D., \& Meyer-Lindenberg, A. (2018). Machine learning for precision psychiatry: Opportunities and challenges. Biological Psychiatry, 3, 223-230. https://doi.org/10.1016/j.bpsc.2017.11.007

Bzdok, D., Varoquaux, G., Grisel, O., Eickenberg, M., Poupon, C., \& Thirion, B. (2016). Formal models of the network co-occurrence underlying mental operations. PLoS Computational Biology, 12, e1004994. https://doi.org/10.1371/journal.pcbi.1004994

Bzdok, D., \& Yeo, B. T. T. (2017). Inference in the age of big data: Future perspectives on neuroscience. Neurolmage, 14(155), 549-564.

Bzdok, D., \& loannidis, J. P. A., (2019). Exploration, inference and prediction in neuroscience and biomedicine. Trend in Neurosciences, 42, 251-262.

Bzdok, D., Nichols, T.E., Smith, S.M., (2019). Towards algorithmic analytics for large-scale datasets. Nature Machine Intelligence, in press.

Calhoun, V. D., Adali, T., Pearlson, G. D., \& Pekar, J. J. (2001). A method for making group inferences from functional MRI data using independent component analysis. Human Brain Mapping, 14(3), 140-151.

Carrión, R. E., Demmin, D., Auther, A. M., McLaughlin, D., Olsen, R., Lencz, T., ... Cornblatt, B. A. (2016). Duration of attenuated positive and negative symptoms in individuals at clinical high risk: Associations with risk of conversion to psychosis and functional outcome. Journal of Psychiatric Research, 81, 95-101. https://doi.org/10.1016/j. jpsychires.2016.06.021

Chai, X. J., Castanon, A. N., Ongur, D., \& Whitfield-Gabrieli, S. (2012). Anticorrelations in resting state networks without global signal regression. Neurolmage, 59(2), 1420-1428. https://doi.org/10.1016/j. neuroimage.2011.08.048

Charlson, F. J., Ferrari, A. J., Santomauro, D. F., Diminic, S., Stockings, E., Scott, J. G., ... Whiteford, H. A. (2018). Global epidemiology and burden of Schizophrenia: Findings from the global burden of disease study 2016. Schizophrenia Bulletin, 44, 1195-1203. https://doi.org/10. 1093/schbul/sby058

Chekroud, A. M., Lane, C. E., \& Ross, D. A. (2017). Computational psychiatry: Embracing uncertainty and focusing on individuals, not averages. Biological Psychiatry, 82(6), e45-e47. https://doi.org/10.1016/j. biopsych.2017.07.011

Chennubhotla, C., \& Jepson, A. (2001). Sparse PCA. Extracting multi-scale structure from data. Proceedings of the 8th IEEE International Conference on Computer Vision, 1, pp. 641-647.

Chong, H. Y., Siew, L. T., Wu, D. B. C., Kotirum, S., Chiou, C. F., \& Chaiyakunapruk, N. (2016). Global economic burden of schizophrenia: A systematic review. Neuropsychiatric Disease and Treatment, 12, 357-373. https://doi.org/10.2147/NDT.S96649

Cole, M. W., Bassett, D. S., Power, J. D., Braver, T. S., \& Petersen, S. E. (2014). Intrinsic and task-evoked network architectures of the human brain. Neuron, 83(1), 238-251. https://doi.org/10.1016/j.neuron. 2014.05.014

Connolly, F. H., \& Gittleson, N. L. (1971). The relationship between delusions of sexual change and olfactory and gustatory hallucinations in schizophrenia. British Journal of Psychiatry, 119(551), 443-444.

Costafreda, S. G., Brammer, M. J., David, A. S., \& Fu, C. H. (2008). Predictors of amygdala activation during the processing of emotional stimuli: A meta-analysis of 385 PET and fMRI studies. Brain Research Reviews, 58(1), 57-70.

Couture, S. M., Penn, D. L., \& Roberts, D. L. (2006). The functional significance of social cognition in Schizophrenia: A review. Schizophrenia Bulletin, 32, S44-S63.

Curcic-Blake, B., Ford, J. M., Hubl, D., Orlov, N. D., Sommer, I. E., Waters, F., ... Aleman, A. (2017). Interaction of language, auditory and memory brain networks in auditory verbal hallucinations. Progress in Neurobiology, 148, 1-20. https://doi.org/10.1016/j.pneurobio.2016.11.002

Dansereau, C., Benhajali, Y., Risterucci, C., Pich, E. M., Orban, P., Arnold, D., \& Bellec, P. (2017). Statistical power and prediction accuracy in multisite resting-state fMRI connectivity. Neurolmage, 149, 220-232. https://doi.org/10.1016/j.neuroimage.2017.01.072

de la Fuente-Sandoval, C., Favila, R., Gomez-Martin, D., Pellicer, F., \& Graff-Guerrero, A. (2010). Functional magnetic resonance imaging response to experimental pain in drug-free patients with schizophrenia. Psychiatry Research, 183(2), 99-104. https://doi.org/10.1016/j. pscychresns.2010.05.003

Derrfuss, J., \& Mar, R. A. (2009). Lost in localization: the need for a universal coordinate database. Neuroimage, 48(1), 1-7.

Derntl, B., Finkelmeyer, A., Toygar, T. K., Hulsmann, A., Schneider, F., Falkenberg, D. I., \& Habel, U. (2009). Generalized deficit in all core components of empathy in schizophrenia. Schizophrenia Research, 108 (1-3), 197-206. https://doi.org/10.1016/j.schres.2008.11.009

Derntl, B., Finkelmeyer, A., Voss, B., Eickhoff, S. B., Kellermann, T., Schneider, F., \& Habel, U. (2012). Neural correlates of the core facets of empathy in schizophrenia. Schizophrenia Research, 136(1-3), 70-81. https://doi.org/10.1016/j.schres.2011.12.018

Dhindsa, R. S., \& Goldstein, D. B. (2016). From genetics to physiology at last. Nature, 530, 162-163.

Donath, W. E., \& Hofman, A. J. (1973). Lower bounds for the partitioning of graphs. IBM Journal of Research and Development, 17, 420-425. 
Dworkin, R. H. (1994). Pain insensitivity in schizophrenia: A neglected phenomenon and some implications. Schizophrenia Bulletin, 20(2), 235-248.

Efron, B. (2012). Large-scale inference: Empirical Bayes methods for estimation, testing, and prediction (Vol. 1). Cambridge, UK: Cambridge University Press.

Efron, B., \& Tibshirani, R. J. (1994). An introduction to the bootstrap. Boca Raton, FL, USA: CRC Press.

Eickhoff, S. B., Bzdok, D., Laird, A. R., Kurth, F., \& Fox, P. T. (2012). Activation likelihood estimation meta-analysis revisited. Neurolmage, 59(3), 2349-2361.

Eickhoff, S. B., \& Etkin, A. (2016). Going beyond finding the "lesion": A path for maturation of neuroimaging. American Journal of Psychiatry, 173(3), 302-303. https://doi.org/10.1176/appi.ajp.2015.15101350

Eickhoff, S. B., Laird, A. R., Grefkes, C., Wang, L. E., Zilles, K., \& Fox, P. T. (2009). Coordinate-based activation likelihood estimation metaanalysis of neuroimaging data: A random-effects approach based on empirical estimates of spatial uncertainty. Human Brain Mapping, 30(9), 2907-2926. https://doi.org/10.1002/hbm.20718

Eickhoff, S. B., Nichols, T. E., Laird, A. R., Hoffstaedter, F., Amunts, K., Fox, P. T., ... Eickhoff, C. R. (2016). Behavior, sensitivity, and power of activation likelihood estimation characterized by massive empirical simulation. Neurolmage, 137, 70-85. https://doi.org/10.1016/j.neuroimage. 2016.04.072

Eickhoff, S. B., Thirion, B., Varoquaux, G., \& Bzdok, D. (2015). Connectivity-based parcellation: Critique and implications. Human Brain Mapping, 36, 4771-4792. https://doi.org/10.1002/hbm.22933

Elert, E. (2014). Searching for schizophrenia's roots. Nature, 508, S2-S3.

Ellison-Wright, I., Glahn, D. C., Laird, A. R., Thelen, S. M., \& Bullmore, E. (2008). The anatomy of first-episode and chronic schizophrenia: An anatomical likelihood estimation meta-analysis. American Journal of Psychiatry, 165(8), 1015-1023. https://doi.org/10.1176/appi.ajp. 2008.07101562

Erickson, M. A., Ruffle, A., \& Gold, J. M. (2016). A meta-analysis of mismatch negativity in Schizophrenia: From clinical risk to disease specificity and progression. Biological Psychiatry, 79(12), 980-987. https:// doi.org/10.1016/j.biopsych.2015.08.025

Fett, A. K., Viechtbauer, W., Dominguez, M. D., Penn, D. L., van Os, J., \& Krabbendam, L. (2011). The relationship between neurocognition and social cognition with functional outcomes in schizophrenia: A metaanalysis. Neuroscience and Biobehavioral Reviews, 35(3), 573-588. https://doi.org/10.1016/j.neubiorev.2010.07.001

Finn, E. S., Shen, X., Scheinost, D., Rosenberg, M. D., Huang, J., Chun, M. M., ... Constable, R. T. (2015). Functional connectome fingerprinting: Identifying individuals using patterns of brain connectivity. Nature Neuroscience, 18(11), 1664-1671. https://doi.org/10.1038/nn.4135

Fletcher, P. C., \& Frith, C. D. (2009). Perceiving is believing: A Bayesian approach to explaining the positive symptoms of schizophrenia. Nature Reviews Neuroscience, 10(1), 48-58.

Forbes, N. F., Carrick, L. A., McIntosh, A. M., \& Lawrie, S. M. (2009). Working memory in schizophrenia: A meta-analysis. Psychological Medicine, 39, 889-905.

Fox, D. F., \& Raichle, M. E. (2007). Spontaneous fluctuations in brain activity observed with functional magnetic resonance imaging. Nature Reviews Neuroscience, 8, 700-711.

Fox, P. T., Laird, A. R., Fox, S. P., Fox, P. M., Uecker, A. M., Crank, M., ... Lancaster, J. L. (2005). BrainMap taxonomy of experimental design: Description and evaluation. Human Brain Mapping, 25(1), 185-198. https://doi.org/10.1002/hbm.20141

Fox, P. T., \& Lancaster, J. L. (1994). Neuroscience on the net. Science, 266, 994-996.

Fox, P. T., \& Lancaster, J. L. (2002). Opinion: Mapping context and content: The BrainMap model. Nature Reviews Neuroscience, 3(4), 319-321. https://doi.org/10.1038/nrn789 nrn789 [pii].

Gabrieli, J. D., Ghosh, S. S., \& Whitfield-Gabrieli, S. (2015). Prediction as a humanitarian and pragmatic contribution from human cognitive neuroscience. Neuron, 85(1), 11-26. https://doi.org/10.1016/j.neuron. 2014.10.047

Gelman, A., \& Hill, J. (2006). Data analysis using regression/hierarchical models: Cambridge University Press.

Glahn, D. C., Laird, A. R., Ellison-Wright, I., Thelen, S. M., Robinson, J. L., Lancaster, J. L., ... Fox, P. T. (2008). Meta-analysis of gray matter anomalies in schizophrenia: Application of anatomic likelihood estimation and network analysis. Biological Psychiatry, 64(9), 774-781. https://doi.org/10.1016/j.biopsych.2008.03.031

Glasser, M. F., Coalson, T., Robinson, E., Hacker, C., Harwell, J., Yacoub, E., ... Jenkinson, M. (2015). A multi-modal parcellation of human cerebral cortex. Nature, 536, 171-1.

Green, M. F., Bearden, C. E., Cannon, T. D., Fiske, A. P., Hellemann, G. S., Horan, W. P., ... Nuechterlein, K. H. (2012). Social cognition in schizophrenia, part 1: Performance across phase of illness. Schizophrenia Bulletin, 38(4), 854-864. https://doi.org/10.1093/schbul/sbq171

Green, M. F., Horan, W. P., \& Lee, J. (2015). Social cognition in schizophrenia. Nature Reviews Neuroscience, 16(10), 620-631. https://doi.org/10. 1038/nrn4005

Hastie, T., Tibshirani, R., \& Friedman, J. (2001). The elements of statistical learning. Heidelberg, Germany: Springer Series in Statistics.

Hegarty, J. D., Baldessarini, R. J., Tohen, M., Waternaux, C., \& Oepen, G. (1994). One hundred years of schizophrenia: A meta-analysis of the outcome literature. The American Journal of Psychiatry, 151(10), 1409-1416.

Honea, R., Crow, T. J., Passingham, D., \& Mackay, C. E. (2005). Regional deficits in brain volume in schizophrenia: A meta-analysis of voxelbased morphometry studies. American Journal of Psychiatry, 162(12), 2233-2245. https://doi.org/10.1176/appi.ajp.162.12.2233

Huys, Q. J. M., Maia, T. V., \& Frank, M. J. (2016). Computational psychiatry as a bridge from neuroscience to clinical applications. Nature Neuroscience, 19(3), 404-413.

Hvarinen, A. (1999). Fast and robust fixed-point algorithms for independent component analysis. IEEE Transactions on Neural Networks, 10(3), 626-634.

Jardri, R., Pouchet, A., Pins, D., \& Thomas, P. (2011). Cortical activations during auditory verbal hallucinations in schizophrenia: A coordinatebased meta-analysis. American Journal of Psychiatry, 168(1), 73-81. https://doi.org/10.1176/appi.ajp.2010.09101522

Javitt, D. C. (2009). When doors of perception close: Bottom-up models of disrupted cognition in schizophrenia. Annual Review of Clinical Psychology, 5, 249-275. https://doi.org/10.1146/annurev.clinpsy.032408.153502

Javitt, D. C., \& Freedman, R. (2015). Sensory processing dysfunction in the personal experience and neuronal machinery of schizophrenia. American Journal of Psychiatry, 172(1), 17-31. https://doi.org/10.1176/appi. ajp.2014.13121691

Javitt, D. C., \& Sweet, R. A. (2015). Auditory dysfunction in schizophrenia: Integrating clinical and basic features. Nature Reviews Neuroscience, 16 (9), 535-550. https://doi.org/10.1038/nrn4002

Johnson, S. C. (1967). Hierarchical clustering schemes. Psychometrika, 32 (3), 241-254.

Kanwisher, N. (2010). Functional specificity in the human brain: A window into the functional architecture of the mind. Proceedings of the National Academy of Sciences of the United States of America, 107(25), 11163-11170. https://doi.org/10.1073/pnas.1005062107

Khalsa, S. S., Adolphs, R., Cameron, O. G., Critchley, H. D., Davenport, P. W., Feinstein, J. S., ... Paulus, M. P. (2017). Interoception and mental health: A roadmap. Biological Psychiatry: Cognitive Neuroscience and Neuroimaging, 3, 501-513. https://doi.org/10.1016/j.bpsc. 2017.12.004

Kohler, C. G., Walker, J. B., Martin, E. A., Healey, K. M., \& Moberg, P. J. (2010). Facial emotion perception in schizophrenia: A meta-analytic review. Schizophrenia Bulletin, 36(5), 1009-1019. https://doi.org/10. 1093/schbul/sbn192

Kuhn, M., \& Johnson, K. (2013). Applied predictive modeling (Vol. 26). New York, USA: Springer. 
Kurtz, M. M., \& Richardson, C. L. (2012). Social cognitive training for schizophrenia: A meta-analytic investigation of controlled research. Schizophrenia Bulletin, 38(5), 1092-1104. https://doi.org/10.1093/ schbul/sbr036

Laird, A. R., Eickhoff, S. B., Fox, P. M., Uecker, A. M., Ray, K. L., Saenz, J. J., Jr., ... Fox, P. T. (2011). The BrainMap strategy for standardization, sharing, and meta-analysis of neuroimaging data. BMC Research Notes, 4(1), 349.

Laird, A. R., Eickhoff, S. B., Kurth, F., Fox, P. M., Uecker, A. M., Turner, J. A., ... Fox, P. T. (2009). ALE meta-analysis workflows via the BrainMap database: Progress towards a probabilistic functional brain atlas. Frontiers in Neuroinformatics, 3, 23. https://doi.org/10.3389/ neuro.11.023.2009

Laird, A. R., Eickhoff, S. B., Li, K., Robin, D. A., Glahn, D. C., \& Fox, P. T. (2009). Investigating the functional heterogeneity of the default mode network using coordinate-based meta-analytic modeling. Journal of Neuroscience, 29(46), 14496-14505.

Laird, A. R., Fox, P. M., Eickhoff, S. B., Turner, J. A., Ray, K. L., McKay, D. R., ... Fox, P. T. (2011). Behavioral interpretations of intrinsic connectivity networks. Journal of Cognitive Neuroscience, 23(12), 4022-4037.

Lee, J., \& Park, S. (2005). Working memory impairments in schizophrenia: A meta-analysis. Journal of Abnormal Psychology, 114(4), 599-611. https://doi.org/10.1037/0021-843x.114.4.599

Lewandowski, K. E., DePaola, J., Camsari, G. B., Cohen, B. M., \& Ongur, D. (2009). Tactile, olfactory, and gustatory hallucinations in psychotic disorders: A descriptive study. Annals of the Academy of Medicine, Singapore, 38(5), 383-385.

Li, H., Chan, R. C., McAlonan, G. M., \& Gong, Q. Y. (2010). Facial emotion processing in schizophrenia: A meta-analysis of functional neuroimaging data. Schizophrenia Bulletin, 36(5), 1029-1039. https://doi.org/10. 1093/schbul/sbn190

Lim, A., Hoek, H. W., Deen, M. L., \& Blom, J. D. (2016). Prevalence and classification of hallucinations in multiple sensory modalities in schizophrenia spectrum disorders. Schizophrenia Research, 176(2-3), 493-499. https://doi.org/10.1016/j.schres.2016.06.010

Llorca, P. M., Pereira, B., Jardri, R., Chereau-Boudet, I., Brousse, G., Misdrahi, D., ... de Chazeron, I. (2016). Hallucinations in schizophrenia and Parkinson's disease: An analysis of sensory modalities involved and the repercussion on patients. Scientific Reports, 6, 38152. https:// doi.org/10.1038/srep38152

Lloyd, S. (1957). Least squares quantization in PCM. Published 1982 in IEEE Transactions on Information Theory, Technical Reports 28, 128-137.

Louppe, G. (2014). Understanding random forests: From theory to practice. arXiv preprint arXiv:1407.7502.

Louppe, G., Wehenkel, L., Sutera, A., \& Geurts, P. (2013). Understanding variable importances in forests of randomized trees. Advances in Neural Information Processing Systems, pp. 431-439.

Lu, H., Zuo, Y., Gu, H., Waltz, J. A., Zhan, W., Scholl, C. A., ... Stein, E. A. (2007). Synchronized delta oscillations correlate with the resting-state functional MRI signal. Proceedings of the National Academy of Sciences of the United States of America, 104(46), 18265-18269. https://doi. org/10.1073/pnas.0705791104

McCarthy-Jones, S., Smailes, D., Corvin, A., Gill, M., Morris, D. W., Dinan, T. G., ... Dudley, R. (2017). Occurrence and co-occurrence of hallucinations by modality in schizophrenia-spectrum disorders. Psychiatry Research, 252, 154-160. https://doi.org/10.1016/j.psychres.2017.01.102

McCleery, A., Lee, J., Fiske, A. P., Ghermezi, L., Hayata, J. N., Hellemann, G. S., ... Knowlton, B. J. (2016). Longitudinal stability of social cognition in schizophrenia: A 5-year follow-up of social perception and emotion processing. Schizophrenia Research, 176(2), 467-472.

McGrath, J., Saha, S., Chant, D., \& Welham, J. (2008). Schizophrenia: A concise overview of incidence, prevalence, and mortality. Epidemiologic Reviews, 30, 67-76. https://doi.org/10.1093/epirev/mxn001

Moberg, P. J., McGue, C., Kanes, S. J., Roalf, D. R., Balderston, C. C., Gur, R. E., ... Turetsky, B. I. (2007). Phenylthiocarbamide (PTC) perception in patients with schizophrenia and first-degree family members:
Relationship to clinical symptomatology and psychophysical olfactory performance. Schizophrenia Research, 90(1-3), 221-228. https://doi. org/10.1016/j.schres.2006.11.014

Moberg, P. J., Roalf, D. R., Balderston, C. C., Kanes, S. J., Gur, R. E., \& Turetsky, B. I. (2005). Phenylthiocarbamide perception in patients with schizophrenia and first-degree family members. American Journal of Psychiatry, 162(4), 788-790. https://doi.org/10.1176/appi.ajp.162. 4.788

Modinos, G., Costafreda, S. G., van Tol, M. J., McGuire, P. K., Aleman, A., \& Allen, P. (2013). Neuroanatomy of auditory verbal hallucinations in schizophrenia: A quantitative meta-analysis of voxel-based morphometry studies. Cortex, 49(4), 1046-1055. https://doi.org/10.1016/j. cortex.2012.01.009

Murphy, K., Birn, R. M., Handwerker, D. A., Jones, T. B., \& Bandettini, P. A. (2009). The impact of global signal regression on resting state correlations: Are anti-correlated networks introduced? Neurolmage, 44(3), 893-905. https://doi.org/10.1016/j.neuroimage.2008.09.036

Namiki, C., Hirao, K., Yamada, M., Hanakawa, T., Fukuyama, H., Hayashi, T., \& Murai, T. (2007). Impaired facial emotion recognition and reduced amygdalar volume in schizophrenia. Psychiatry Research, 156(1), 23-32. https://doi.org/10.1016/j.pscychresns.2007.03.004

Nanetti, L., Cerliani, L., Gazzola, V., Renken, R., \& Keysers, C. (2009). Group analyses of connectivity-based cortical parcellation using repeated kmeans clustering. Neurolmage, 47(4), 1666-1677. https://doi.org/10. 1016/j.neuroimage.2009.06.014

Nelson, S. M., Dosenbach, N. U., Cohen, A. L., Wheeler, M. E., Schlaggar, B. L., \& Petersen, S. E. (2010). Role of the anterior insula in task-level control and focal attention. Brain Structure and Function, 214 (5-6), 669-680. https://doi.org/10.1007/s00429-010-0260-2

Nielsen, J. A., Zielinski, B. A., Fletcher, P. T., Alexander, A. L., Lange, N., Bigler, E. D., ... Anderson, J. S. (2013). Multisite functional connectivity MRI classification of autism: ABIDE results. Frontiers in Human Neuroscience, 7(599), 1-12. https://doi.org/10.3389/fnhum.2013.00599

Ochsner, K. N. (2008). The social-emotional processing stream: Five core constructs and their translational potential for schizophrenia and beyond. Biological Psychiatry, 64(1), 48-61.

Oquendo, M. A., Baca-Garcia, E., Artes-Rodriguez, A., Perez-Cruz, F., Galfalvy, H. C., Blasco-Fontecilla, H., ... Duan, N. (2012). Machine learning and data mining: Strategies for hypothesis generation. Molecular Psychiatry, 17(10), 956-959. https://doi.org/10.1038/mp.2011.173

Owens, A. P., Allen, M., Ondobaka, S., \& Friston, K. J. (2018). Interoceptive inference: From computational neuroscience to clinic. Neuroscience and Biobehavioral Reviews, 90, 174-183. https://doi.org/10.1016/j. neubiorev.2018.04.017

Passingham, R. E., Stephan, K. E., \& Kotter, R. (2002). The anatomical basis of functional localization in the cortex. Nature Reviews Neuroscience, 3 (8), 606-616. https://doi.org/10.1038/nrn893

Pedregosa, F., Varoquaux, G., Gramfort, A., Michel, V., Thirion, B., Grisel, O., ... Dubourg, V. (2011). Scikit-learn: Machine learning in Python. Journal of Machine Learning Research, 12(Oct), 2825-2830.

Poldrack, R. A., \& Yarkoni, T. (2016). From brain maps to cognitive ontologies: Informatics and the search for mental structure. Annual Review of Psychology, 67, 587-612. https://doi.org/10.1146/annurev-psych122414-033729

Rissling, A. J., \& Light, G. A. (2010). Neurophysiological measures of sensory registration, stimulus discrimination, and selection in schizophrenia patients. In Behavioral neurobiology of schizophrenia and its treatment (pp. 283-309). New York, USA: Springer.

Satterthwaite, T. D., Elliott, M. A., Gerraty, R. T., Ruparel, K., Loughead, J., Calkins, M. E., ... Wolf, D. H. (2013). An improved framework for confound regression and filtering for control of motion artifact in the preprocessing of resting-state functional connectivity data. Neurolmage, 64, 240-256. https://doi.org/10.1016/j.neuroimage.2012.08.052

Savla, G. N., Vella, L., Armstrong, C. C., Penn, D. L., \& Twamley, E. W. (2013). Deficits in domains of social cognition in schizophrenia: A 
meta-analysis of the empirical evidence. Schizophrenia Bulletin, 39(5), 979-992. https://doi.org/10.1093/schbul/sbs080

Schneider, F., Habel, U., Reske, M., Kellermann, T., Stocker, T., Shah, N. J., ... Gaebel, W. (2007). Neural correlates of working memory dysfunction in first-episode schizophrenia patients: An fMRI multi-center study. Schizophrenia Research, 89(1-3), 198-210. https://doi.org/10. 1016/j.schres.2006.07.021

Shlens, J. (2014). A tutorial on principal component analysis. arXiv preprint arXiv:1404.1100.

Shmueli, G. (2010). To explain or to predict? Statistical Science, 25, 289-310.

Silva, R. F., Castro, E., Gupta, C. N., Cetin, M., Arbabshirani, M., Potluru, V. K., ... Calhoun, V. D. (2014). The tenth annual MLSP competition: Schizophrenia classification challenge. Paper presented at the Machine Learning for Signal Processing (MLSP), 2014 IEEE International Workshop on.

Smith, S. M., Fox, P. T., Miller, K. L., Glahn, D. C., Fox, P. M., Mackay, C. E., ... Beckmann, C. F. (2009). Correspondence of the brain's functional architecture during activation and rest. PNAS, 106(31), 13040-13045.

Sporns, O. (2014). Contributions and challenges for network models in cognitive neuroscience. Nature Neuroscience, 17(5), 652-660. https:// doi.org/10.1038/nn.3690

Stephan, K. E., Schlagenhauf, F., Huys, Q. J. M., Raman, S., Aponte, E. A., Brodersen, K. H., ... Dolan, R. J. (2017). Computational neuroimaging strategies for single patient predictions. Neurolmage, 145, 180-199.

Stone, M. (1974). Cross-validatory choice and assessment of statistical predictions. Journal of the Royal Statistical Society Series B, 36, 111-147.

Stone, M. (1978). Cross-validation: A review. Statistics: A Journal of Theoretical and Applied Statistics, 9(1), 127-139.

Stubbs, B., Mitchell, A. J., De Hert, M., Correll, C. U., Soundy, A., Stroobants, M., \& Vancampfort, D. (2014). The prevalence and moderators of clinical pain in people with schizophrenia: A systematic review and large scale meta-analysis. Schizophrenia Research, 160(1-3), 1-8. https://doi.org/10.1016/j.schres.2014.10.017

Stubbs, B., Thompson, T., Acaster, S., Vancampfort, D., Gaughran, F., \& Correll, C. U. (2015). Decreased pain sensitivity among people with schizophrenia: A meta-analysis of experimental pain induction studies. Pain, 156 (11), 2121-2131. https://doi.org/10.1097/j.pain.0000000000000304

Sullivan, P. F. (2012). Schizophrenia as a pathway disease. Nature Medicine, 18, 210-211. https://doi.org/10.1038/nm.2670

Tandon, R., Gaebel, W., Barch, D. M., Bustillo, J., Gur, R. E., Heckers, S., ... Carpenter, W. (2013). Definition and description of schizophrenia in the DSM-5. Schizophrenia Research, 150(1), 3-10. https://doi.org/10. 1016/j.schres.2013.05.028

Tavor, I., Parker Jones, O., Mars, R. B., Smith, S. M., Behrens, T. E., \& Jbabdi, S. (2016). Task-free MRI predicts individual differences in brain activity during task performance. Science, 352(6282), 216-220. https://doi.org/10.1126/science.aad8127

Taylor, S. F., Kang, J., Brege, I. S., Tso, I. F., Hosanagar, A., \& Johnson, T. D. (2012). Meta-analysis of functional neuroimaging studies of emotion perception and experience in schizophrenia. Biological Psychiatry, 71 (2), 136-145. https://doi.org/10.1016/j.biopsych.2011.09.007

Thirion, B., Varoquaux, G., Dohmatob, E., \& Poline, J. B. (2014). Which fMRI clustering gives good brain parcellations? Frontiers in Neuroscience, 8, 167. https://doi.org/10.3389/fnins.2014.00167

Thomas, M. L., Green, M. F., Hellemann, G., Sugar, C. A., Tarasenko, M., Calkins, M. E., ... Light, G. A. (2017). Modeling deficits from early auditory information processing to psychosocial functioning in schizophrenia. JAMA Psychiatry, 74(1), 37-46. https://doi.org/10.1001/jamapsychiatry.2016. 2980
Thomas, P., Mathur, P., Gottesman, I. I., Nagpal, R., Nimgaonkar, V. L., \& Deshpande, S. N. (2007). Correlates of hallucinations in schizophrenia: A cross-cultural evaluation. Schizophrenia Research, 92(1-3), 41-49. https://doi.org/10.1016/j.schres.2007.01.017

Tost, H., \& Meyer-Lindenberg, A. (2012). Puzzling over schizophrenia: Schizophrenia, social environment and the brain. Nature Medicine, 18 (2), 211-213. https://doi.org/10.1038/nm.2671

Turkeltaub, P. E., Eickhoff, S. B., Laird, A. R., Fox, M., Wiener, M., \& Fox, P. (2012). Minimizing within-experiment and within-group effects in activation likelihood estimation meta-analyses. Human Brain Mapping, 33 (1), 1-13. https://doi.org/10.1002/hbm.21186

Umbricht, D., \& Krljes, S. (2005). Mismatch negativity in schizophrenia: A meta-analysis. Schizophrenia Research, 76(1), 1-23. https://doi.org/10. 1016/j.schres.2004.12.002

Van Essen, D. C., Anderson, C. H., \& Felleman, D. J. (1992). Information processing in the primate visual system: An integrated systems perspective. Science, 255(5043), 419-423.

van Luxburg, U. (2007). A tutorial on spectral clustering. Statistics and Computing, 17, 395-416.

Waters, F., Collerton, D., Ffytche, D. H., Jardri, R., Pins, D., Dudley, R., ... Larøi, F. (2014). Visual hallucinations in the psychosis spectrum and comparative information from neurodegenerative disorders and eye disease. Schizophrenia Bulletin, 40(Suppl. 4), S233-S245. https://doi. org/10.1093/schbul/sbu036

Weinberger, D. R., \& Radulescu, E. (2016). Finding the elusive psychiatric "lesion" with 21st-century neuroanatomy: A note of caution. American Journal of Psychiatry, 173(1), 27-33. https://doi.org/10.1176/appi.ajp. 2015.15060753

Winkler, A. M., Ridgway, G. R., Douaud, G., Nichols, T. E., \& Smith, S. M. (2016). Faster permutation inference in brain imaging. Neurolmage, 141, 502-516.

Wittchen, H. U., Jacobi, F., Rehm, J., Gustavsson, A., Svensson, M., Jönsson, B., ... Steinhausen, H. C. (2011). The size and burden of mental disorders and other disorders of the brain in Europe 2010. European Neuropsychopharmacology, 21, 655-679. https://doi.org/10. 1016/j.euroneuro.2011.07.018

Wolpert, D. H. (1992). Stacked generalization. Neural Networks, 5(2), 241-260.

Yarkoni, T., Poldrack, R. A., Nichols, T. E., Van Essen, D. C., \& Wager, T. D. (2011). Large-scale automated synthesis of human functional neuroimaging data. Nature Methods, 8(8), 665-670. https://doi.org/10.1038/nmeth.1635

Yeh, C. J., Tseng, Y. S., Lin, Y. R., Tsai, S. Y., \& Huang, T. Y. (2015). Resting-state functional magnetic resonance imaging: The impact of regression analysis. Journal of Neuroimaging, 25(1), 117-123. https://doi.org/10.1111/jon. 12085

Zou, H., Hastie, T., \& Tibshirani, R. (2006). Sparse principal component analysis. Journal of Computational and Graphical Statistics, 15(2), 265-286.

\section{SUPPORTING INFORMATION}

Additional supporting information may be found online in the Supporting Information section at the end of this article.

How to cite this article: Karrer TM, Bassett DS, Derntl B, et al. Brain-based ranking of cognitive domains to predict schizophrenia. Hum Brain Mapp. 2019;40:4487-4507. https:// doi.org/10.1002/hbm.24716 